\title{
Stabilité d'une cellule de gabion sous poids propre
}

\author{
Stability analysis of a cofferdam cell
}

\author{
L. DORMIEUX \\ Laboratoire de Mécanique des Solides* \\ C. DELAURENS \\ Ecole Nationale des Ponts et Chaussées *
}

Rev. Franç. Géotech. n 55, pp. 47-61 (avril 1991)

\section{Résumé}

Les gabions cellulaires sont des ouvrages fréquemment rencontrés en site aquatique où ils sont utilisés pour la réalisation de batardeaux et de travaux de soutènement. En s'appuyant sur la théorie du calcul à la rupture, on présente une analyse de la stabilité d'une cellule de gabion au terme de la phase de construction, sous l'action des forces de pesanteur.

On fournit un encadrement du poids volumique extrême du matériau de remblai en fonction des capacités de résistance de ce dernier et de la résistance à l'arrachement des serrures de palplanches. On examine successivement le cas d'un remblai constitué d'un matériau cohérent et celui d'un remblai constitué d'un matériau frottant.

L'approche proposée prend en compte le caractère tridimensionnel de la géométrie de la structure. Elle permet une relecture critique de méthodes classiques de dimensionnement.

\section{Abstract}

Cellular cofferdams are frequently used in marine environment for construction in the dry. The stability of a cell subjected to gravity forces at the end of the filling stage is analysed using the yield design theory.

Lower and upper bound estimates of the critical unit weight of the fill material are derived. They depend on the material's yield criterion and on the maximum allowable interlock tension. The case of a purely frictional fill material and the case of a purely cohesive fill material are both considered.

The proposed approach accounts for the threedimensional geometry of the problem. A critical review of classical design methods is also presented.

- Ecole Polytechnique, 91128 Palaiseau Cedex.

* Le Central 2, La Courtine, 93167 Noisy-le-Grand. 


\section{INTRODUCTION}

Les gabions cellulaires sont des ouvrages de génie civil fréquemment rencontrés dans les travaux en site aquatique. Ils sont utilisés dans la réalisation d'ouvrages de soutènement, de batardeaux ou de ducs d'Albe. Ils sont constitués d'un assemblage de cellules identiques juxtaposées et solidarisées les unes aux autres. Chaque cellule se compose d'une enceinte cylindrique formée de palplanches et remplie par un matériau de remblai.

La tenue de ces ouvrages est assurée simultanément par les capacités de résistance du matériau de remblai qui est contraint à la périphérie par le rideau de palplanches, et par celles du rideau lui-même. Elle résulte de l'interaction entre remblai et rideau, dont Tanalyse mécanique de la cellule doit rendre compte. Dans le calcul de ces ouvrages, il faut donc prendre en considération à la fois les risques d'instabilité externe (poinçonnement, basculement ou glissement d'ensemble) et ceux d'instabilité interne (rupture de la structure mixte)

L'état actuel des connaissances dans le domaine des gabions est caractérisé par la diversité des théories qui ont été proposées. Dans certains cas, la comparaison des règles de dimensionnement auxquelles elles conduisent révèle des différences significatives. A ce jour, aucune approche n'a su s'imposer.

Dans le sens d'une analyse mécanique plus satisfaisante, une première voie consiste à tenter de résoudre numériquement le problème en déplacements par la méthode des éléments finis, ce qui requiert la donnée d'une loi de comportement pour les matériaux d'enceinte et de remblai (CLOUGH et al., 1987). Dans cette direction, la nature tridimensionnelle de la géométrie de la structure constitue une difficulté importante.

De plus, dans la pratique, la connaissance du comportement dont on dispose tant sur le matériau de remblai que sur les interfaces se limite souvent à la description des capacités de résistance, c'est-à-dire la donnée d'un critère de rupture. La théorie du calcul à la rupture fournit l'outil qui permet d'exploiter complètement ce type d'information dans un cadre mécanique rigoureux

On se propose à présent d'en illustrer l'emploi sur le problème de la stabilité d'une cellule élémentaire soumise à son poids propre, à l'exclusion de tout autre effort.

\section{PRINCIPE DE LA THÉORIE DU CALCUL A LA RUPTURE}

\subsection{Approches statique et cinématique}

Ce paragraphe décrit succinctement les idées maîtresses du calcul à la rupture. Pour une présentation détaillée de cette théorie, on pourra se reporter aux travaux de J. SALENÇON (1983, 1990).

On considère un domaine matériel $\Omega$ soumis à une sollicitation caractérisée par la donnée d'un nombre fini de $\mathrm{N}$ paramètres de chargement. L'objet du calcul à la rupture est la détermination ou l'encadrement du domaine $\mathcal{K}$ de $\mathbb{R}^{\mathrm{N}}$ constitué par les chargements dits potentiellement supportables. Par définition, il s'agit des chargements qu'il est possible d'équilibrer par un champ de contraintes compatible en tout point du domaine $\Omega$ avec les capacités de résistance du matériau constitutif. La frontière de $\nVdash$ définit l'ensemble des chargements extrêmes.

En s'appuyant sur cette définition, on dispose donc en premier lieu d'une méthode directe, dite statique puisqu'elle est basée sur la construction de champs de contraintes, qui permet d'identifier un sous-ensemble du domaine $\mathscr{K}$ recherché. Cette méthode statique conduit ainsi à approcher $\mathcal{K}$ par l'intérieur.

Pour obtenir un encadrement de $\mathcal{K}$, on a recours à une méthode duale de la précédente, basée sur la construction de champs de vitesses virtuelles, et dite à ce titre cinématique. Elle consiste à comparer, dans un champ de vitesses $\mathrm{v}$ cinématiquement admissible quelconque du problème, la valeur de la puissance des efforts extérieurs appliqués à $\Omega$, notée $P_{\text {ext }}(v)$, avec la puissance que peut opposer le domaine $\Omega$ au mouvement virtuel envisagé, du fait des capacités de résistance du matériau qui le constitue. Cette dernière, dite puissance résistante maximale, est définie de la manière suivante :

$\mathrm{P}(\underline{\mathrm{v}})=\int_{\Omega} \pi(\underline{\mathrm{x}}, \underline{\mathrm{d}}(\underline{\mathrm{x}})) \mathrm{d} \Omega+\int_{\Sigma} \pi(\underline{\mathrm{x}}, \underline{\mathrm{n}}(\underline{\mathrm{x}}),[\underline{\mathrm{v}}(\underline{\mathrm{x}})]) \mathrm{d} \Sigma$

où $\mathrm{d}$ désigne le tenseur taux de déformations associé à $\underline{v} ; \Sigma$, les surfaces de discontinuité du champ $\underline{v}$, si elles existent ; $[\underline{v}(\underline{x})]$, la discontinuité du champ $\underline{v}$ au point $\underline{x}$ de $\Sigma ; \underline{n}(\underline{x})$, la normale unitaire à $\Sigma$ au point $x$

Les fonctions $\pi$ introduites dans l'expression de $\mathrm{P}(\mathrm{v})$ sont entièrement définies par la donnée dans $\mathbb{R}^{6}$ du domaine $\mathrm{G}(\mathrm{x})$ des contraintes admissibles au point $\mathrm{x}$ dans le cadre du critère utilisé, supposé convexe. Elles ont la forme suivante:

$$
\begin{aligned}
& \pi(\underline{x}, \underline{\mathrm{d}}(\underline{\mathrm{x}}))=\sup (\underline{\underline{\sigma}}(\underline{\mathrm{x}}): \underline{\mathrm{d}}(\underline{\mathrm{x}}), \underline{\sigma}(\underline{\mathrm{x}}) \in \mathrm{G}(\underline{\mathrm{x}})) \\
& \pi(\underline{\mathrm{x}}, \underline{\mathrm{n}}(\underline{\mathrm{x}}),[\underline{\mathrm{v}}(\underline{\mathrm{x}})])=\sup ([\underline{\mathrm{v}}(\underline{\mathrm{x}})], \underline{\underline{\sigma}}(\underline{\mathrm{x}}), \underline{\mathrm{n}}(\underline{\mathrm{x}}), \underline{\underline{\sigma}} \underline{\underline{\mathrm{x}})} \in \mathrm{G}(\underline{\mathrm{x}}))
\end{aligned}
$$

Pour tout champ de vitesses $v$ cinématiquement admissible, le théorème cinématique fournit une condition nécessaire de stabilité à vérifier par la sollicitation sous la forme de l'inégalité $P_{\text {ext }}(v) \leq P(v)$.

Lorsque la sollicitation est définie dans le cadre d'un mode de chargement à $\mathrm{N}$ paramètres notés $\mathrm{Q}=$ $\left(Q_{i}\right)_{1 \leq i \leq n}$, la puissance des efforts extérieurs dañs le champ $v$ prend la forme d'un produit scalaire :

$$
Q_{\text {ext }}(\underline{\mathrm{v}})=\underline{\mathrm{Q}} \cdot \dot{q}(\underline{\mathrm{v}})
$$

qui fait apparaître le vecteur $\underline{q}(\underline{v})$, appelé vitesse de déformation du système. Dans l'espace de dimension $\mathrm{N}$ des paramètres de chargements, l'inégalité 
$P_{\text {ext }}(\underline{v}) \leq \mathrm{P}(\underline{v})$ délimite deux demi-espaces séparés par l'hyperplan d'équation $\underline{Q} \cdot \underline{q}(\underline{v})=P(\underline{v})$. Le demiespace $\underline{\mathrm{Q}} \cdot \underline{\mathrm{q}}(\underline{\mathrm{v}})>\mathrm{P}(\underline{\mathrm{v}})$ correspond à des chargements qui ne sont pas supportés par le domaine $\Omega$ (voir fig. 1).

Pour chaque choix d'un mécanisme de rupture, c'està-dire pour chaque champ de vitesses $\underline{v}$ cinématiquement admissible, le demi-espace $\underline{Q} \cdot \underline{q}(\underline{v}) \leq P(\underline{v})$ fournit une approche dite par l'extérieur, c'est-à-dire un sur-ensemble du convexe $\mathcal{K}$ des chargements potentiellement supportables. Dans la pratique, on examine en général plusieurs mécanismes et l'on détermine l'intersection de ces demi-espaces. On observera que l'application du théorème cinématique n'est fructueuse que dans le cas où la fonctionnelle $\mathrm{P}(\mathrm{v})$ est bornée, ce qui assujettit le champ des vitesses virtuelles $\mathrm{v}$ à vérifier des conditions dépendant du critère de rūpture adopté.

\subsection{LIEN AVEC LES MÉTHODES DE DIMENSIONNEMENT CLASSIQUES}

Les méthodes de dimensionnement classiques d'une cellule sous l'action des forces de pesanteur au terme de la phase de remplissage sont de type statique (voir par exemple NAVDOCKS DM, 1986). Le principe en est rappelé brièvement ci-après en se plaçant, pour simplifier, dans le cas d'une cellule cylindrique circulaire posée sur un substratum rigide en l'absence d'eau (fig. 2a).

En négligeant le frottement entre le rideau et le remblai, on admet tout d'abord que la verticale (axe $\mathrm{Oz}$ ) est, en tout point, une direction principale du tenseur des contraintes et que le rideau est soumis, de la part du remblai, à un champ de pressions $\mathrm{p}(\mathrm{z})$, fonction de la profondeur dans la cellule. La première hypothèse fixe la valeur de la contrainte verticale $\sigma_{z z}$, qui vaut donc $\gamma_{r} z$, où $\gamma_{\mathrm{r}}$ désigne le poids volumique du matériau de remblai.

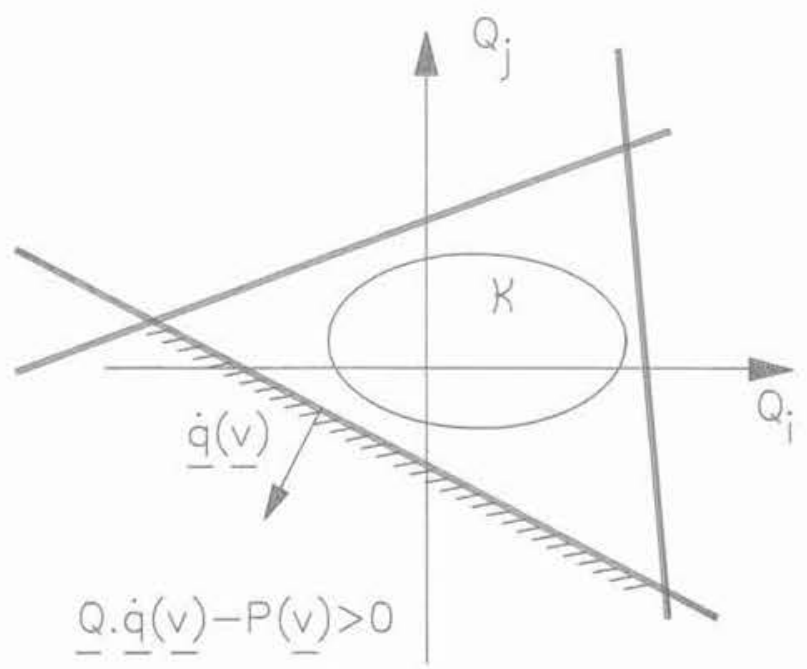

Fig. 1. - Approche cinématique.

Fig. 1, - Kinematic approach.

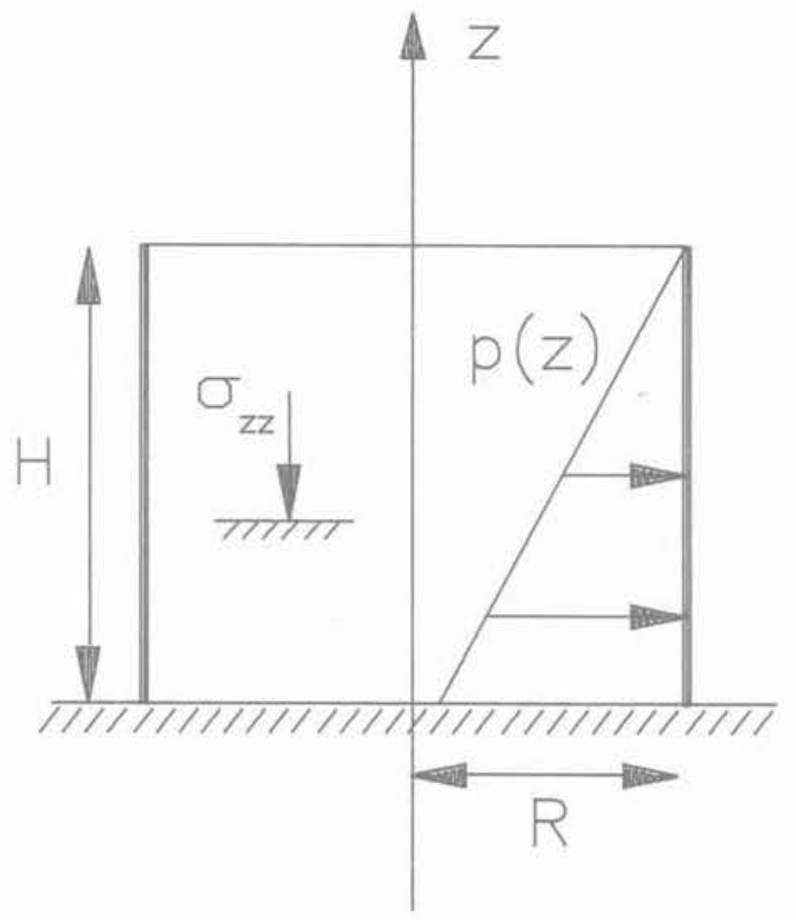

(a)

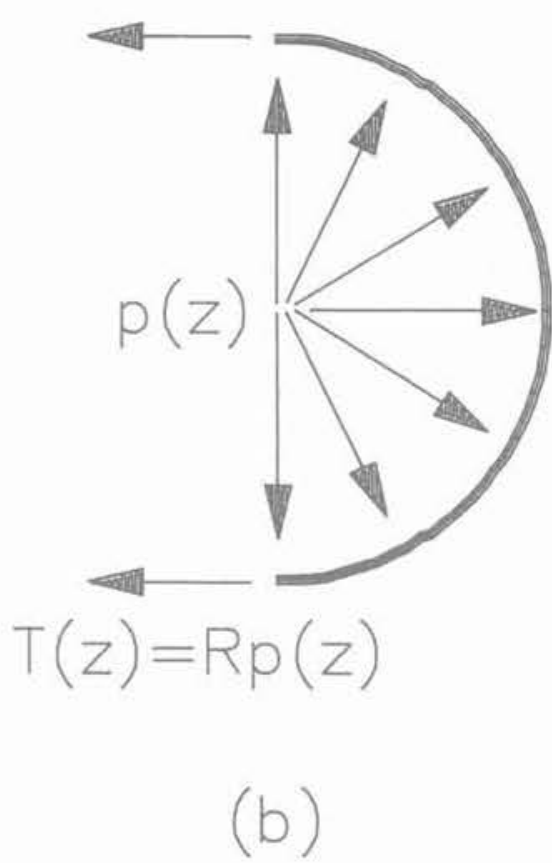

Fig. 2. - Méthodes classiques de dimensionnement. Fig. 2. - Classical design methods.

On introduit alors usuellement un coefficient $\mathrm{K}$, dit de poussée des terres, défini classiquement par l'égalité $\mathrm{p}(\mathrm{z})=\mathrm{K}\left|\sigma_{z z}\right|$. La traction $\mathrm{T}(\mathrm{z})$ dans les serrures par unité de longueur est déterminée par des considérations sur l'équilibre d'une coque mince cylindrique circulaire de rayon $\mathrm{R}$ sous pression, qui conduisent à $\mathrm{T}(\mathrm{z})=\mathrm{p}(\mathrm{z}) \mathrm{R}$ (formule dite * des chaudronniers $»$, (fig. 2b). La traction limite $T_{0}$ par unité de longueur 
de serrures étant donnée, on peut enfin calculer un coefficient de sécurité $\mathrm{F}$ vis-à-vis du risque de rupture par arrachement des serrures au moyen de la relation $F=\inf _{z}\left(T_{0} / T(z)\right)=T_{0} /\left(K \gamma_{r} R H\right)$, dans laquelle $\mathrm{H}$ désigne la hauteur de remblai.

Avec la notation $\gamma_{\mathrm{r}}^{*}=\frac{1}{\mathrm{~K}}\left(\mathrm{~T}_{\mathrm{o}} / \mathrm{RH}\right)$, on remarquera que $F$ n'est autre que le rapport $\gamma_{\mathrm{r}}^{*} / \gamma_{\mathrm{r}}$. En accord avec la définition d'un coefficient de sécurité, $\gamma_{i}^{*}$ apparait donc comme le poids volumique maximal supportable par la structure. Certains aménagements de la démarche précédente sont proposés lorsque les palplanches sont fichées dans le substratum.

La difficulté d'emploi du coefficient de sécurité $F$ ou de la grandeur $\gamma_{r}^{*}$ réside dans le fait que le choix du coefficient $\mathrm{K}$ est arbitraire ou empirique. Ainsi, les valeurs attribuées à $\mathrm{K}$ dans la littérature varient selon les auteurs. Par exemple, SCHNEEBELI et al. (1957) proposent de fixer $\mathrm{K}$ à la valeur du cofficient de poussé limite $\mathrm{K}_{\mathrm{a}}$. LACROIX et al,, (1970) et NAVDOCKS DM (1986) recommandent plutôt la valeur $\mathrm{K}=0,4$, ce qui conduit selon les cas à un coefficient de sécurité plus ou moins élevé qu'avec $\mathrm{K}=\mathrm{K}_{\mathrm{a}}$. Il est donc légitime de s'interroger sur le bien-fondé de la terminologie de * coefficient de sécurité $»$.

Une reformulation du raisonnement précédent dans le cadre de l'approche par l'intérieur du calcul à la rupture sera présentée aux paragraphes 4.1. et 5.1., respectivement pour un remblai cohérent ou frottant. On verra qu'elle conduit à des conditions suffisantes de stabilité potentielle.

\section{LE PROBLÈME POSÉ}

\subsection{Modélisation de la géométrie}

Du point de vue géométrique, on modélise l'enceinte de palplanches par un tube circulaire de hauteur $H$, de rayon intérieur $R$, de rayon extérieur $R+e$, et d'axe $\mathrm{Oz}$ (voir fig. $3 \mathrm{a}$ ), avec la condition $e \ll R$. Le tube est constitué de $\mathrm{n}$ palplanches identiques. La palplanche $n^{\circ} i$ occupe dans cette enceinte l'espace compris entre les plans verticaux passant par l'axe du cylindre et formant avec le plan $\mathrm{Oxz}$ respectivement

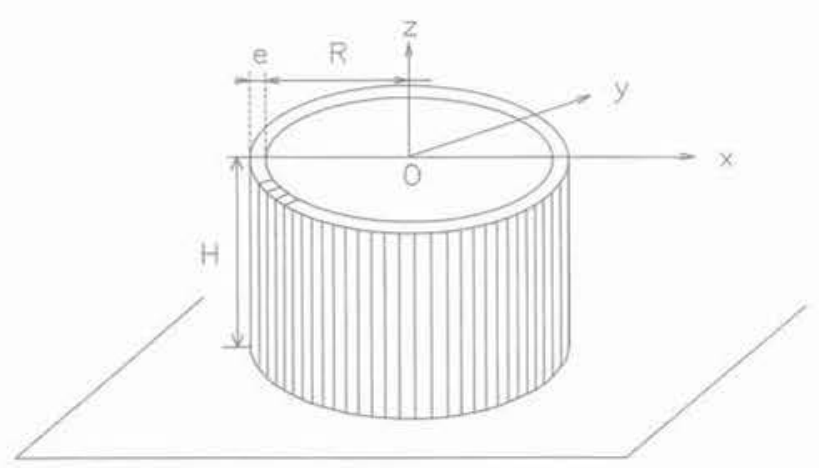

Fig. 3a. - Géométrie de la cellule.

Fig. $3 a$, - Geometry of the cell.

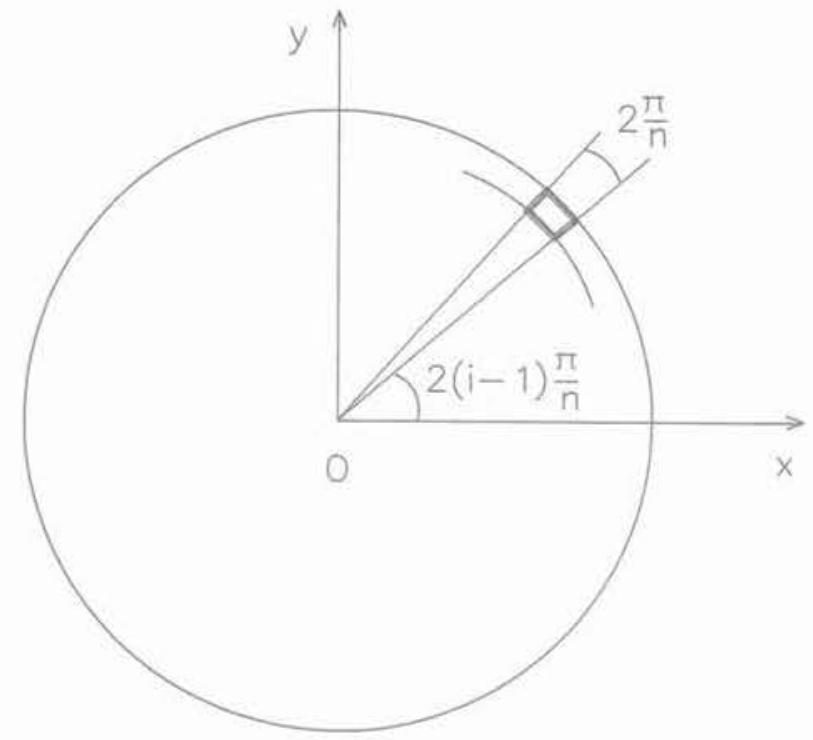

Fig. $3 b .-$ Repérage de la palplanche $n^{\circ} i$.

Fig. $3 b$ - Geometry of the sheetpile assemblage.

les angles $2(i-1) \frac{\pi}{n}$ et $2 i \frac{\pi}{n}$ (voir fig. $3 b$ ). Le remblai occupe à l'intérieur de l'enceinte un cylindre circulaire de rayon $\mathrm{R}$ et de hauteur $\mathrm{H}$. L'ensemble de la cellule est posé sur le substratum rigide.

\subsection{Le chargement}

On s'intéresse à la stabilité de la cellule ainsi décrite au terme de la phase de construction. Le système mécanique considéré est constitué par le substratum et la cellule. Les faces latérale et supérieure de la cellule ainsi que le domaine du plan $\mathrm{z}=-\mathrm{H}$ en dehors du disque $r \leq R+e$ sont libres de contraintes. Les forces de gravité sont donc les seules forces extérieures. Elles sont caractérisées par les poids volumiques $\gamma_{\mathrm{s}}, \gamma_{\mathrm{r}}$ et $\gamma_{\mathrm{a}}$, respectivement du substratum, du matériau de remblai et de l'acier.

\subsection{Modélisation des capacités de résistance}

On utilise dans ce document la convention de signe consistant à compter positivement les contraintes de traction.

La description des capacités de résistance est faite au moyen de critères de rupture se rapportant d'une part aux matériaux constituant le remblai, les palplanches et le substratum, d'autre part aux différentes interfaces.

Deux grandes classes de remblais seront examinées successivement aux paragraphes 4 et 5 . Ils sont désignés respectivement par les qualificatifs "purement cohérent * et « purement frottant ».

\section{Remblai purement cohérent}

Le critère de rupture du matériau de remblai est un critère de Tresca défini par la cohésion $C_{r}$ :

$$
\mathrm{f}_{\mathrm{r}} \underline{\underline{(\sigma)}}=\sup _{\mathrm{i}, \mathrm{p}}\left(\sigma_{\mathrm{i}}-\sigma_{\mathrm{j}}-2 \mathrm{C}_{\mathrm{r}}\right) \leq 0
$$




\section{Remblai purement frottant}

Le critère de rupture du matériau de remblai est un critère de Mohr-Coulomb. On note $\phi_{\mathrm{r}}$ Pangle de frottement interne :

$$
f_{r} \underline{\underline{(\sigma)}}=\sigma_{1}-\sigma_{3}+\left(\sigma_{1}+\sigma_{3}\right) \sin \phi_{\mathrm{r}}
$$

où $\sigma_{1}$ et $\sigma_{3}$ désignent respectivement les valeurs principales majeure et mineure du tenseur des contraintes $\underline{\sigma}$.

\section{Substratum et palplanches}

Les capacités de résistance des matériaux constituant le substratum et les palplanches seront supposées infinies.

\section{Les interfaces}

- Le critère d'interface entre les palplanches $n^{\circ} i$ et $n^{\circ} i+1$ est destiné à modéliser les capacités de résistance des serrures par lesquelles ces palplanches sont solidarisées. L'interface occupe géométriquement un rectangle de hauteur $\mathrm{H}$ et de largeur $e$, situé dans le plan par $O$ et parallèle aux vecteurs:

$$
\underline{e}_{x}=\underline{e}_{x} \cos \frac{2 i \pi}{n}+\underline{e}_{y} \sin \frac{2 i \pi}{n}
$$

et $\underline{e}_{z}$ (voir fig. 4). L'expression du critère est la suivante :

$$
f_{p}(\underline{I})=\sup \left(\underline{T} \cdot \underline{e}_{\theta}-T_{0} / e\right) \leq 0
$$

La grandeur $\mathrm{T}_{0}$ (en $\mathrm{N} \cdot \mathrm{m}^{-1}$ ) est une donnée caractérisant la résistance de la serrure. Le vecteur $\mathrm{T}$ désigne le vecteur-contrainte agissant sur l'interface. Le critère adopté consiste donc à imposer une limite supérieure à la contrainte normale, sans restriction (à défaut de données) sur les composantes tangentielles de ce vecteur.

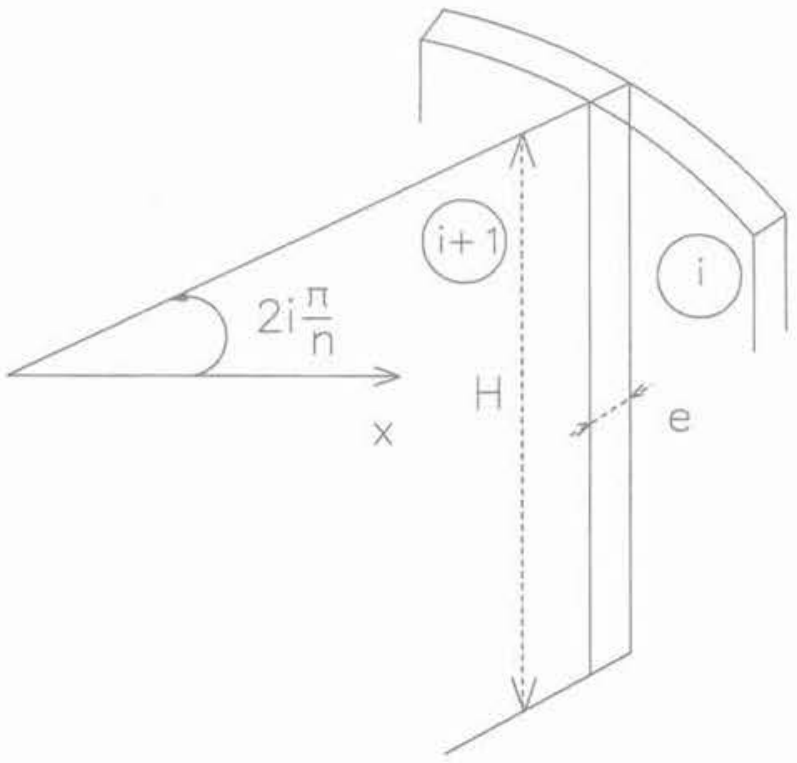

Fig. 4. - Interface entre les palplanches $n^{\circ} i$ et $i+1$. Fig. 4, - Interface between sheet piles $n^{\circ} i$ and $i+1$.
- Interface entre le substratum et les palplanches:

$$
f_{s p}(\underline{T})=\sup \left(|\underline{\tau}|, \sigma_{n}\right) \leq 0
$$

- Interface entre le substratum et le remblai :

$$
\mathrm{f}_{\mathrm{rs}}(\underline{\mathrm{T}})=\sup \left(|\underline{\tau}|, \sigma_{\mathrm{n}}\right) \leq 0
$$

- Interface entre le remblai et les palplanches

$$
f_{\text {tp }}(\underline{T})=\sup \left(|\underline{\tau}|, \sigma_{n}\right) \leq 0
$$

où $\underline{\tau}=\underline{T}-(\underline{T} \cdot \underline{n}) \underline{n}$ et $\sigma_{\mathrm{n}}=\underline{T} \cdot \underline{n}$ désignent les composantes dū vecteur-contrainte ${ }^{-} T$, respectivement tangentielle et perpendiculaire à l'interface de normale $\mathrm{n}$. Ces trois derniers critères correspondent à une interface lisse, la composante $\tau$ du vecteur-contrainte étant assujettie à être nulle, êt sans résistance à la traction, la composante normale $\sigma_{n}$ du même vecteur devant être négative (voir fig. 5).

\subsection{Remarques générales}

On observera que l'hypothèse consistant à admettre que les capacités de résistance de l'acier constituant les palplanches sont infinies impose pour la suite que la cinématique de chaque palplanche prise isolément soit définie par un mouvement rigidifiant pour lequel la déformation est nulle en tout point. Tout autre choix conduirait à une contribution infinie des palplanches à la puissance résistante maximale de la structure.

L'hypothèse identique formulée pour le substratum indique que les mécanismes de rupture de la cellule impliquant le substratum (poinçonnement, glissement circulaire, etc.) ne sont pas pertinents, pour les mêmes raisons.

Le choix d'interfaces toutes lisses et sans résistance à la traction est manifestement conservatif, au sens où il consiste à négliger la mobilisation d'un frottement ou d'une cohésion entre les divers constituants de la structure. Il est motivé notamment par la difficulté rencontrée dans l'évaluation des caractéristiques des interfaces. La fonction $\pi(\mathrm{V})$ de la discontinuité au niveau d'une telle interface est définie de la manière suivante :

$$
\begin{aligned}
& \pi(\underline{\mathrm{V}})=+\infty \text { si } \underline{\mathrm{V}} \cdot \underline{\mathrm{n}}=\mathrm{V}_{\mathrm{n}}<0 \\
& \pi(\underline{\mathrm{V}})=0 \quad \text { si } \underline{\mathrm{V}} \cdot \underline{\mathrm{n}}=\mathrm{V}_{\mathrm{n}} \geq 0
\end{aligned}
$$

Ce résultat signifie que les cinématiques relatives des divers constituants de la structure doivent être construites sans interpénétration (voir fig, 5).

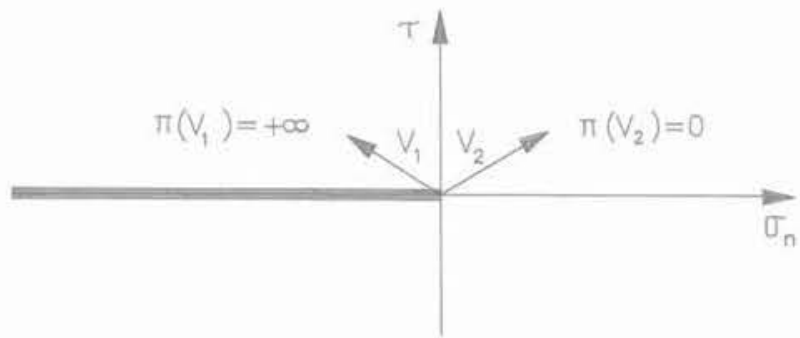

Fig. 5. - Interface lisse sans résistance à la traction. Fig. 5, - Smooth interface with zero tensile strength. 
Il peut être tentant de réduire la définition du système mécanique à la cellule elle-même. L'interface cellulesubstratum devient alors une partie de la frontière du système, sur laquelle il faut définir des conditions aux limites, en imposant par exemple une condition de contact unilatéral sans frottement. Ce procédé présente l'avantage de permettre l'économie des critères $f_{s p}$ et $f_{r p}$. De plus, dans les approches statiques, la construction des champs de contraintes se limite alors évidemment à la cellule. Toutefois, on observera que l'emploi de la méthode cinématique est alors restreint dans la pratique à des champs de vitesses assurant le contact bilatéral $\left(\mathrm{V}_{\mathrm{z}}=0\right)$ sur le plan $\mathrm{z}=-\mathrm{H}$. En particulier, un mécanisme tel que celui décrit au paragraphe 4.2.2., dans lequel la composante verticale de la vitesse des palplanches est non nulle en $\mathrm{z}=-\mathrm{H}$, ne pourrait être utilisé avec une telle définition du système. Par ailleurs, celle-ci n'est plus adaptée au problème posé dans le cas où les capacités de résistance du substratum ne sont plus supposées infinies.

\section{4. ÉTUDE DE LA STRUCTURE COHÉRENTE}

\subsection{Approche statique}

On a vu que la frontière du système étudié est libre de contraintes. Les données en efforts du problème sont donc caractérisées, a priori, par les poids volumiques $\gamma_{a}, \gamma_{r}$ et $\gamma_{s}$, respectivement de l'acier, du sol de remblai et du matériau constituant le substratum. Il s'agit donc d'approcher le domaine $\mathcal{K}$ de $\mathbb{R}^{3}$ des triplets $\left(\gamma_{a}, \gamma_{r}, \gamma_{s}\right)$ potentiellement supportables.

Pour développer une approche statique de la frontière de $\pi$, c'est-à-dire déterminer un sous-ensemble de $\mathcal{K}$, il convient en premier lieu de rappeler l'ensemble des conditions auxquelles est soumis un champ de contraintes statiquement admissible avec les données en efforts du problème ainsi posé.

Equations de champ :

- dans le remblai

$(\mathrm{r} \in[0, \mathrm{R}] ; z \in[-\mathrm{H}, 0]): \operatorname{div} \underline{\underline{\sigma}}-\gamma_{\mathrm{r}} \underline{e}_{z}=\underline{0}$

- dans l'enceinte :

$(r \in[R, R+e] ; z \in[-H, 0]): \operatorname{div} \underline{\underline{\sigma}}-\gamma_{\mathrm{a}} \underline{e_{z}}=\underline{0}$

- dans le substratum :

$$
(z<-H): \operatorname{div} \underline{\underline{\sigma}}-\gamma_{\mathrm{s}} \underline{e}_{z}=\underline{0}
$$

Conditions aux limites :

- face latérale de la cellule:

$$
(\mathrm{r}=\mathrm{R}+\mathrm{e} ; \mathrm{z} \in[-\mathrm{H}, 0]): \underline{\underline{\sigma}} \cdot \underline{e}_{x}=\underline{0}
$$

- face supérieure de la cellule :

$$
(\mathrm{r} \in[0, \mathrm{R}+\mathrm{e}] ; \mathrm{z}=0): \underline{\sigma} \cdot \underline{e_{z}}=\underline{0}
$$

- domaine plan :

$$
\mathrm{z}=-\mathrm{H} ; \mathrm{r}>\mathrm{R}+\mathrm{e}: \underline{\underline{\sigma}} \cdot \underline{\mathrm{e}}_{3}=\underline{0}
$$

On se propose de construire une solution des équations précédentes $(12 \mathrm{a}, \mathrm{b}, \mathrm{c})$ et $(13 \mathrm{a}, \mathrm{b}, \mathrm{c})$, possé- dant la symétrie de révolution, telle qu'en tout point les directions principales du tenseur des contraintes soient les directions radiale, orthoradiale et verticale, et qui soit de plus compatible avec les capacités de résistance des matériaux et des interfaces.

En raison des hypothèses sur la symétrie et l'orientation des directions principales du champ de contraintes à construire, les équations de champ se réduisent à :

$$
\begin{gathered}
\frac{\partial \sigma_{\pi}}{\partial r}+\frac{\sigma_{\pi r}-\sigma_{\theta \theta}}{r}=0 \\
\frac{\partial \sigma_{z z}}{\partial z}=\left\{\begin{array}{l}
\gamma_{a} \text { dans les palplanches } \\
\gamma_{r} \text { dans le remblai } \\
\gamma_{s} \text { dans le substratum }
\end{array}\right.
\end{gathered}
$$

avec les conditions aux limites suivantes:

$$
\begin{array}{ll}
(\forall z \in[-H, 0]) & \sigma_{\mathrm{rr}}(\mathrm{r}=\mathrm{R}+\mathrm{e}, \mathrm{z})=0 \\
(\forall \mathrm{r} \in[0, \mathrm{R}+\mathrm{e}]) & \sigma_{\mathrm{zz}}(\mathrm{r}, \mathrm{z}=0)=0 \\
(\forall \mathrm{r} \in[\mathrm{R}+\mathrm{e},+\infty[) & \sigma_{\mathrm{zz}}(\mathrm{r}, \mathrm{z}=\mathrm{H})=0
\end{array}
$$

Enfin, les composantes $\sigma_{t r}$ et $\sigma_{z z}$ sont assujetties à être continues au passage des interfaces remblai/palplanches et cellule/substratum. On observera que les équations (14b) et (15) déterminent complètement les variations de la composante $\sigma_{z z}$ dans le système:

$$
\begin{array}{rlrl}
\underline{z>0} ; & r<R & \sigma_{z z}=\gamma_{\mathrm{r}} \mathrm{R} \\
\mathrm{r} \in[\mathrm{R}, \mathrm{R}+\mathrm{e}] & \sigma_{z z}=\gamma_{\mathrm{a}} \mathrm{z} \\
\underline{\mathrm{z}<0 ;} ; & \mathrm{r}<\mathrm{R} & \sigma_{\mathrm{zz}}=\gamma_{\mathrm{s}}(\mathrm{z}+\mathrm{H})-\gamma_{\mathrm{r}} \mathrm{H} \\
& \mathrm{r} \in[\mathrm{R}, \mathrm{R}+\mathrm{e}] & \sigma_{\mathrm{zz}}=\gamma_{\mathrm{s}}(\mathrm{z}+\mathrm{H})-\gamma_{\mathrm{a}} \mathrm{H} \\
\mathrm{r}>\mathrm{R}+\mathrm{e} & \sigma_{\mathrm{zz}}=\gamma_{\mathrm{s}}(\mathrm{z}+\mathrm{H})
\end{array}
$$

Le problème différentiel défini par (14) et (15) comporte donc 2 fonctions inconnues $\sigma_{\pi}$ et $\sigma_{\theta \theta}$ des arguments $r$ et $z$, qu'il s'agit de construire en sorte que le champ de contraintes soit compatible avec les capacités de résistances des matériaux mis en jeu.

Les capacités de résistance de l'interface cellule/sub. stratum sont satisfaites indépendamment du choix de $\sigma_{r r}$ et $\sigma_{\theta \theta}$, en raison du signe $(<0)$ de $\sigma_{z z}$ en $z=-H$. De plus, celles du matériau constituant le substratum étant infinies, la construction du champ $\underline{\underline{\sigma}}$ peut en fait être limitée à la cellule. En effet, pour tout choix de $\sigma_{\pi r}$ et $\sigma_{\theta \theta}$ dans la cellule, l'existence d'un prolongement statiquement admissible de $\sigma$ dans le substratum est assurée. Pour s'en convaincrē, il suffit de vérifier que le champ (discontinu) défini cidessous dans le domaine $z<-H$, répond, entre autres, à la question :

$$
\underline{\underline{\sigma}}=S(z)\left[\underline{e}_{y} \otimes \underline{e}_{y}+\underline{e}_{\theta} \otimes \underline{e}_{\theta}\right]+\sigma_{z z} \underline{e}_{y} \otimes \underline{e}_{z}
$$

où la fonction $S(z)$ peut être choisie arbitrairement. Un premier résultat de stabilité peut alors être obtenu simplement au moyen du champ de contraintes uniaxial suivant :

$$
\begin{array}{ll}
\underline{\underline{\sigma}}=\gamma_{\mathrm{r}} \mathrm{z} \underline{\underline{e}}_{2} \otimes \underline{e}_{2} & \text { dans le remblai } \\
\underline{\underline{\sigma}}=\gamma_{\mathrm{a}} \mathrm{z} \underline{e}_{2} \otimes \underline{e}_{3} & \text { dans l'enceinte }
\end{array}
$$


dont la discontinuité en $\mathrm{r}=\mathrm{R}$ est statiquement admissible. Introduisant le paramètre de chargement adimensionnel $\mathrm{k}=\gamma_{\mathrm{r}} \mathrm{H} / \mathrm{C}_{r}$, on observe que ce champ est compatible avec les capacités de résistance de la cellule dès lors que $\mathrm{k} \leq 2$. Notant $\mathrm{k}^{+}$la borne supérieure des valeurs de $\mathrm{k}$ potentiellement supportables, on dispose donc de l'inégalité :

$$
\mathrm{k}^{+} \geq 2
$$

Notant de même $\gamma_{r}^{+}$la borne supérieure des valeurs de $\gamma_{\mathrm{r}}$ potentiellement supportables, (19) peut être réexprimée de manière équivalente par:

$$
\gamma_{r}^{+} \geq 2 \mathrm{C}_{r} / \mathrm{H}
$$

On examine maintenant la stabilité potentielle de cellules pour lesquelles $\mathrm{H} \geq 2 \mathrm{C}_{\mathrm{r}} / \gamma_{\mathrm{r}}$. On commence par définir le champ $\underline{\sigma}$ dans le remblai de la manière suivante :

$$
\begin{gathered}
z>-2 C_{r} / \gamma_{r}: \underline{\sigma}=\gamma_{\mathrm{r}} z \underline{e}_{z} \otimes \underline{e}_{z} \\
-H<z<-2 C_{r} / \gamma_{r}: \underline{\sigma}= \\
\gamma_{\mathrm{r}}\left\{\left(z+2 C_{r} / \gamma_{r}\right)\left[\underline{e}_{\gamma} \otimes \underline{e}_{s}+\underline{e}_{\theta} \otimes \underline{e}_{\theta}\right]+z \underline{e}_{z} \otimes \underline{e}_{3}\right\}
\end{gathered}
$$

La donnée de deux expressions distinctes de $\sigma$ selon les valeurs de $z$ est rendue nécessaire par l'absence de résistance à la traction de l'interface remblaipalplanches. Il est immédiat de vérifier qu'il est compatible avec les capacités de résistance du matériau de remblai. Dans l'enceinte, on distingue de même selon la valeur de $z$ :

$$
z>-2 C_{r} / \gamma_{r}: \underline{\underline{\sigma}}=\gamma_{\mathrm{a}} \mathrm{z} \underline{e}_{3} \otimes \underline{e}_{3}
$$

$-\mathrm{H}<z<-2 \mathrm{C}_{\mathrm{r}} / \gamma_{\mathrm{r}}$; on recherche $\underline{g}$ sous la forme suivante :

$$
\begin{array}{r}
\underline{\underline{\sigma}}=\left(\mathrm{a}(\mathrm{z})+\mathrm{b}(\mathrm{z}) / \mathrm{r}^{2}\right) \underline{e}_{3} \otimes \underline{e}_{3}+ \\
\left(\mathrm{a}(\mathrm{z})-\mathrm{b}(\mathrm{z}) / \mathrm{r}^{2}\right) \underline{e}_{\theta} \otimes \underline{e}_{\theta}+\gamma_{\mathrm{a}} \mathrm{z} \underline{e}_{z} \otimes \underline{e}_{z}
\end{array}
$$

Les valeurs de $a(z)$ et $b(z)$ sont obtenues en écrivant la condition de continuité de $\sigma_{\pi}$ en $r=R$, et la condition aux limites (13a) en $r=R+e$. Il vient :

$$
\begin{aligned}
& a(z)=\frac{-b(z)}{(R+e)^{2}} \\
& b(z)=\frac{\gamma_{r}\left(z+2 C_{r} / \gamma_{r}\right)}{1 / R^{2}-1 /(R+e)^{2}}
\end{aligned}
$$

La condition de compatibilité de $\underline{\underline{\sigma}}$ avec les capacités de résistance de l'interface entre palplanches s'écrit :

$$
\begin{aligned}
& \sup \sigma_{\theta \theta} \leq \mathrm{T}_{0} / e \\
& z \in[-\mathrm{H}, 0] \\
& \mathrm{r} \in[\mathrm{R}, \mathrm{R}+\mathrm{e}]
\end{aligned}
$$

soit encore, au premier ordre près en $\frac{e}{R}$ :

$$
\mathrm{k} \leq 2+\frac{\mathrm{T}_{\mathrm{o}}}{\mathrm{RC}_{\mathrm{r}}}
$$

qui exprime une condition suffisante de stabilité potentielle de la cellule élémentaire sous son poids propre. L'inégalité (24a) peut être exprimée sous la forme ciaprès qui est équivalente :

$$
\mathrm{k}^{+} \geq 2+\frac{\mathrm{T}_{\mathrm{o}}}{\mathrm{RC}_{\mathrm{r}}}
$$

Cette nouvelle minoration de $\mathrm{k}^{+}$présente, par rapport à (19), l'avantage de faire intervenir la résistance du rideau de palplanches. Elle peut également être exprimée en fonction du poids volumique extrême $\gamma_{\mathrm{r}}^{+}$:

$$
\gamma_{\mathrm{r}}^{+} \geq 2 \mathrm{C}_{\mathrm{r}} / \mathrm{H}+\mathrm{T}_{0} /(\mathrm{RH})
$$

On observera que la condition obtenue est indépendante de $\gamma_{\mathrm{a}}$ et $\gamma_{\mathrm{s}}$. Ceci doit être attribué au fait que les capacités de résistance de l'acier et du substratum sont infinies, ainsi que la résistance en compression de l'interface palplanches/substratum. Géométriquement, cela signifie que le domaine de l'espace $\left(\gamma_{\mathrm{a}}\right.$, $\left.\gamma_{r}, \gamma_{s}\right)$ défini par:

$$
0 \leq \gamma_{\mathrm{r}} \leq 2 \mathrm{C}_{\mathrm{r}} / \mathrm{H}+\mathrm{T}_{\mathrm{o}} / \mathrm{RH}
$$

constitue une approche par l'intérieur dans $\mathbb{R}^{3}$ de $\mathcal{K}$.

L'approche statique qui vient d'être développée peut être aisément généralisée au cas où le substratum possède une cohésion $\mathrm{C}_{5}$ non infinie. En utilisant le champ de contraintes défini en (17) avec:

$$
S(z)=\gamma_{s}(z+H)-\sup \left(\gamma_{a}, \gamma\right) H / 2
$$

il est facile de vérifier que la stabilité potentielle du système est assurée si l'on ajoute à (25) la condition :

$$
\sup \left(\gamma_{a}, \gamma_{p}\right) \leq 4 C_{s} / H
$$

\subsection{Approche cinématique}

Pour lanalyse de la structure élémentaire soumise aux seules forces de gravité, il est naturel de rechercher des mécanismes de rupture pour lesquels la composante orthoradiale de la vitesse soit nulle dans le remblai : $V_{\theta}=0$. On a déjà indiqué que le mouvement virtuel de chacune des palplanches est assujetti à être rigidifiant. De même, pour que la contribution du remblai à la puissance résistance maximale soit finie, le champ de vitesses virtuelles doit y respecter la * condition d'incompressibilité », soit :

$$
\operatorname{tr} \underline{\underline{\mathrm{d}}}=\mathrm{V}_{\mathrm{r}, \mathrm{r}}+\mathrm{V}_{\mathrm{r}} / \mathrm{r}+\mathrm{V}_{\mathrm{z}, 2}=0
$$

On se propose d'exploiter l'idée simplificatrice consistant à explorer, parmi l'ensemble des couples $\left(V_{r}\right.$. $V_{z}$ ) solutions de cette équation, ceux pour lesquels $V_{2}$ est indépendant de $r$. On pose ainsi $V_{z}=f(z)$ En d'autres termes, il s'agit de champs de vitesses dans lesquels les sections de la cellule par les plans horizontaux restent planes. Les capacités de résistance en compression de l'interface remblai/substratum n'étant pas bornées, il convient d'observer dès maintenant que $\mathrm{f}(-\mathrm{H}) \geq 0$ est une condition nécessaire pour que la contribution de l'interface substratum/remblai à la puissance résistante maximale soit finie. Elle est alors nulle. Intuitivement, il convient de choisir la fonction $f$ en sorte que soit maximisée la puissance des forces de gravité. Cette remarque conduit à retenir une fonction négative et par conséquent à imposer $f(-H)=0$. Le choix de $f$ étant fixé, celui de 
la composante radiale $\mathrm{V}_{t}$ de la vitesse virtuelle est imposé par la condition d'incompressibilité qui fournit :

$$
V_{t, r}+V_{r} / r=-f^{\prime}(z)
$$

dont la solution générale en $V_{s}$ est de la forme:

$$
V_{r}=\frac{A}{r}-\frac{r}{2} f^{\prime}(z)
$$

La constante A doit être annulée en sorte que la contribution du remblai à la puissance résistante maximale de l'ensemble de la structure soit finie. On trouve donc :

$$
\begin{aligned}
& V_{r}=-\frac{r}{2} f^{\prime}(Z) \\
& V_{\theta}=0 \\
& V_{z}=f(z)
\end{aligned}
$$

La matrice du tenseur taux de déformation d dans la base $\left(\underline{e}_{x}, \underline{e}_{\theta}, \underline{e}_{2}\right)$ est alors la suivante:

$$
\underline{\mathrm{d}}=\left[\begin{array}{ccc}
-\mathrm{f}^{\prime} / 2 & 0 & -\mathrm{rf} \mathrm{f}^{\prime \prime} / 4 \\
0 & -\mathrm{f}^{\prime} / 2 & 0 \\
-\mathrm{rf} \mathrm{f}^{\prime \prime} / 4 & 0 & \mathrm{f}
\end{array}\right]
$$

Pour le matériau de Tresca, la fonction $\pi$ (d) vaut :

$$
\pi(\underline{\underline{d}})=\mathrm{C}_{\mathrm{r}}\left(\left|\mathrm{d}_{1}\right|+\left|\mathrm{d}_{2}\right|+\left|\mathrm{d}_{3}\right|\right)
$$

où les $\mathrm{d}_{i}$ sont les valeurs principales du tenseur $\mathrm{d}$. En utilisant (32), il vient :

$$
\begin{aligned}
& \mathrm{d}_{1}=-\mathrm{f}^{\prime} / 2 \\
& \left.\begin{array}{l}
d_{2} \\
d_{3}
\end{array}\right\}=\frac{1}{4}\left(-f^{\prime} \pm \sqrt{9 f^{\prime} 2+r^{2} f^{\prime \prime}}\right)
\end{aligned}
$$

ce qui conduit à :

$$
\pi(\underline{\underline{d}})=\frac{1}{2} C_{r}\left[\left|f^{\prime}\right|+\sqrt{9 f^{\prime 2}+r^{2} f^{\prime \prime 2}}\right]
$$

qui permet de calculer la contribution du remblai à la puissance résistance maximale. Afin de compléter la description de la cinématique dans l'enceinte de palplanches, il convient à présent de particulariser le choix de la fonction $\mathrm{f}$. Le choix le plus simple d'une fonction $\mathrm{f}$ vérifiant $\mathrm{f}(-\mathrm{H})=0$ est celui d'une fonction affine. On examine ci-après l'application du théorème cinématique au champ de vitesses virtuelles correspondant.

\subsubsection{Champ de vitesses virtuelles $n^{\circ} 1$ :} $f(z)$ affine

\section{- Contribution du remblai à la puissance résis- tante maximale}

La fonction $f$ étant choisie affine, on construit le champ de vitesses dans la cellule élémentaire successivement dans le remblai puis dans l'enceinte de palplanches. Posant $f(z)=-2 \lambda(z+H)$ dans (31), les vitesses virtuelles dans le remblai sont définies en coordonnées cylindriques, pour $r \in[0, R], z \in$ $[-\mathrm{H}, 0], \theta \in[0,2 \pi]$, par :

$$
\begin{aligned}
& V_{t}=\lambda r \\
& V_{\theta}=0 \\
& V_{z}=-2 \lambda(z+H)
\end{aligned}
$$

Une telle cinématique correspond à une transformation homogène et conserve la géométrie de cylindre circulaire d'axe $\mathrm{Oz}$ (voir fig. 6b). Appliquant (35), et notant $\mathrm{P}_{\mathrm{r}}$ la contribution du remblai à la puissance résistance maximale, on obtient immédiatement :

$$
\pi(\underline{\underline{d}})=4 \lambda \mathrm{C}_{\mathrm{r}} \text { et } \mathrm{P}_{\mathrm{r}}=4 \lambda \mathrm{C}_{\mathrm{r}} \pi \mathrm{R}^{2} \mathrm{H}
$$

Par ailleurs, on a vu précédemment que la condition $\mathrm{f}(-\mathrm{H})=0$ assure que la contribution de l'interface remblai/substratum (lisse sans résistance à la traction) à la puissance résistance maximale soit nulle. On définit maintenant les vitesses virtuelles dans l'enceinte de palplanches.

\section{- Cinématique des palplanches}

- Le champ de vitesses dans chaque palplanche est rigidifiant.

- On désigne par $C_{1}$ le point de la palplanche $n^{\circ}$ i de coordonnées cylindriques $\left(r=R, \theta=(2 i-1) \frac{\pi}{n}\right.$, $z=0)$. Le champ de vitesses de la palplanche $n^{\circ} i$ (voir fig. 6a) est une translation colinéaire au

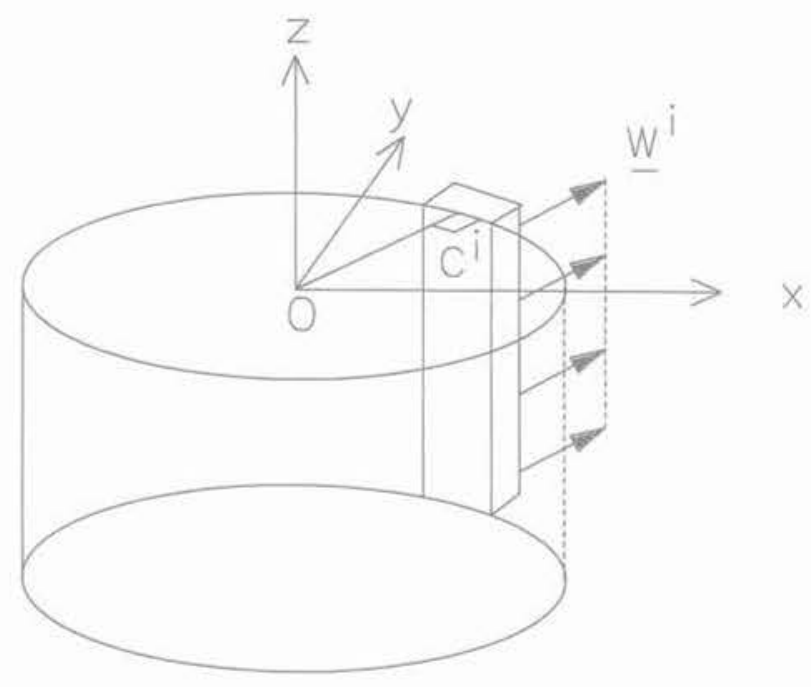

(a) palplanches

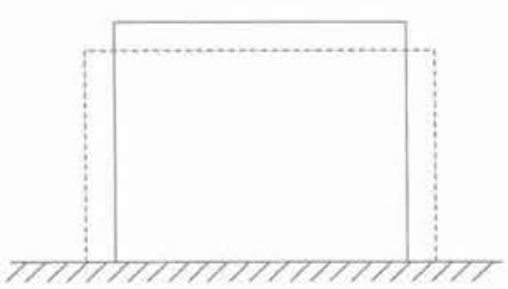

(b) remblai

Fig. 6. - Champ de vitesses virtuelles no 1 . Fig. 6. - Virtual velocity field $n^{\circ}$ ? 
vecteur $O C^{\prime}$. Le vecteur de la translation est défini par :

$$
\begin{aligned}
& \underline{W}^{i}=\frac{\lambda R}{\cos (\pi / n)}\left\{\underline{e}_{x} \cos (2 i-1) \frac{\pi}{n}\right. \\
& \left.+\underline{e}_{y} \sin (2 i-1) \frac{\pi}{n}\right\}
\end{aligned}
$$

Un tel mécanisme peut être interprété comme un éclatement radial de l'enceinte.

- Contribution des palplanches à la puissance résistante maximale

Interface palplanche-remblai

Soit $\mathrm{M}$ le point de coordonnées cylindriques $(r=R$, $\left.\theta=\delta+(2 \mathrm{i}-1) \frac{\pi}{\mathrm{n}}, \mathrm{z}\right)$ de linterface entre la fa-

ce interne de la palplanche $n^{\circ} \mathrm{i}$ et le remblai (voir fig. 7). La discontinuité de vitesse en $M$ vaut :

$$
\begin{aligned}
\underline{\underline{V}}= & \underline{W}^{\dagger}-\lambda\left[\mathrm { R } \left[\underline{e}_{\mathrm{x}} \cos \left((2 \mathrm{i}-1) \frac{\pi}{\mathrm{n}}+\delta\right)\right.\right. \\
& \left.\left.+\underline{e}_{y} \sin \left((2 \mathrm{i}-1) \frac{\pi}{\mathrm{n}}+\delta\right)\right]-2(\mathrm{z}+\mathrm{H}) \underline{e}_{z}\right]
\end{aligned}
$$

En désignant par $\underline{\mathrm{n}}$ le vecteur unitaire radial, normal à l'interface au point $M$, c'est-à-dire :

$$
\begin{aligned}
\underline{n} & =\underline{e}_{x} \cos \left((2 \mathrm{i}-1) \frac{\pi}{\mathrm{n}}+\delta\right) \\
& +\underline{e}_{y} \sin \left((2 \mathrm{i}-1) \frac{\pi}{\mathrm{n}}+\delta\right)
\end{aligned}
$$

on voit que :

$$
\text { [y] } \cdot \underline{n}=\lambda R\left(\frac{\cos \delta}{\cos (\pi / n)}-1\right)
$$

Comme $\delta \in\left[-\frac{\pi}{n},+\frac{\pi}{n}\right]$, le produit scalaire [V] . $\mathrm{n}$ qui représente la composante normale de la discontinuité de vitesses est toujours $\geq 0$. Il résulte de (11b) que la contribution de l'interface palplancheremblai à la puissance résistante maximale est nulle.

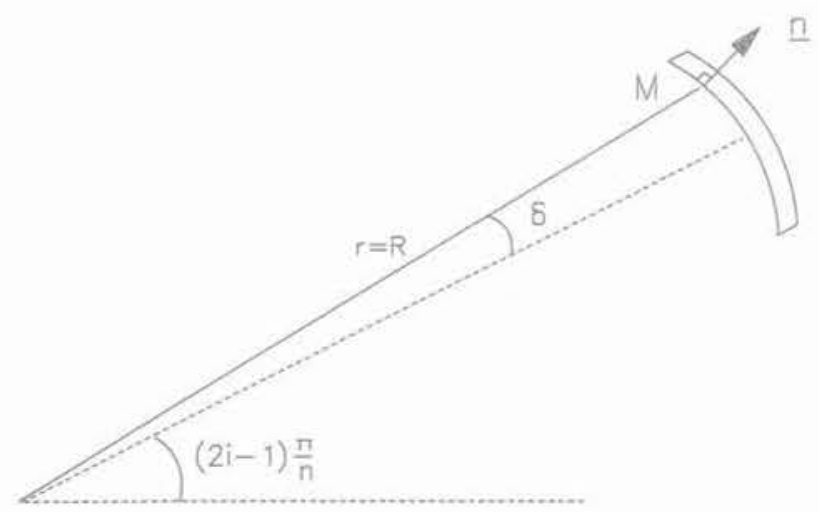

Fig. 7. - Paramétrage de l'interface remblailpalplanche $n^{\circ} i$. Fig. 7. - Definition of parameter $\delta$.

\section{Interfaces entre palplanches}

Il faut maintenant calculer la contribution de la discontinuité de vitesse à l'interface entre les palplanches $n^{\circ} \mathrm{i}$ et $\mathrm{i}+1$. Celle-ci est manifestement orthoradiale :

$$
\begin{aligned}
& \underline{V}^{i+1}-\underline{V}^{i}= \\
& 2 \lambda \operatorname{Rtg}\left(\frac{\pi}{n}\right)\left\{-\underline{e}_{x} \sin (2 i) \frac{\pi}{n}+\underline{e}_{y} \cos (2 i) \frac{\pi}{n}\right\}
\end{aligned}
$$

La fonction $\pi(\underline{V})$ correspond au critère d'interface $\mathrm{f}_{\mathrm{p}}(\mathrm{T})$ définissant la résistance à l'arrachement des palplanches est définie par:

$$
\begin{aligned}
& \pi(\underline{V})=+\infty \text { si } U_{n}<0 \text { ou } U_{t} \neq 0 \\
& \pi(\underline{V})=U_{n} T_{0} / \text { e si } U_{n} \geq 0 \text { et } U_{t}=0
\end{aligned}
$$

On vérifie immédiatement que :

$$
\begin{gathered}
\left(\underline{V}^{i+1}-\underline{V}^{i}\right) \cdot \underline{e}_{y}=\left(\underline{V}^{i+1}-\underline{V}_{i}\right) \cdot \underline{e}_{z}=U_{i}=0 \\
\left(\underline{V}^{i+1}-\underline{V}^{i}\right) \cdot \underline{e}_{\theta}=2 \lambda \operatorname{Rtg}\left(\frac{\pi}{n}\right)
\end{gathered}
$$

Quand $\mathrm{n} \gg 1$, la somme $\mathrm{P}_{\mathrm{p}}$ des contributions des $\mathrm{n}$ interfaces entre palplanches vaut donc:

$$
P_{p}=2 \lambda R_{H} T_{\circ} n t g\left(\frac{\pi}{n}\right)=2 \pi \lambda R_{H} T_{\circ}
$$

\section{- Application du théorème cinématique}

Avant de pouvoir appliquer le théorème cinématique, il reste à calculer la puissance des forces extérieures, qui coïncide avec celles des forces de gravité agissant sur le remblai :

$P_{\text {ext }}=\int_{0}^{R} d r \int_{0}^{2 \pi} r d \theta \int_{-H}^{0}-2 \lambda(z+H) \gamma_{r} d z$

soit :

$$
Q_{\text {ext }}=\gamma_{r} \pi \mathrm{R}^{2} \mathrm{H}^{2} \lambda
$$

La condition nécessaire de stabilité pour la cellule élémentaire résultant de l'application du théorème cinématique à ce mécanisme est donc:

$$
\gamma_{\mathrm{r}} \pi \mathrm{R}^{2} \mathrm{H}^{2} \lambda \leq 4 \lambda \mathrm{C}_{\mathrm{r}} \pi \mathrm{R}^{2} \mathrm{H}+2 \lambda \mathrm{R} \pi \mathrm{HT}_{0}
$$

soit, tous calculs faits :

$$
\mathrm{k}^{+} \leq 4+2\left(\mathrm{~T}_{0} / \mathrm{RC}_{\mathrm{r}}\right)
$$

Le rapport entre le majorant de $\mathrm{k}^{+}$ainsi défini, et le minorant résultant de l'approche statique donné en (24b) est donc exactement 2. On se propose d'affiner l'encadrement qui vient d'être établi en utilisant un deuxième champ de vitesses virtuelles faisant appel à une cinématique plus complexe obtenue en choisissant $f(z)$ de la forme $\alpha\left(z^{2}-H^{2}\right)$. 


\subsubsection{Champ de vitesses virtuelles $n^{\circ} 2$ : $f(z)$ parabolique}

\section{- Contribution du remblai à la puissance résis- tante maximale}

En adoptant une variation parabolique de $V_{2}$ en fonction de $z$ dans (31), on trouve, pour $r \in[0, R]$, $z \in[-H, 0], \theta \in[0,2 \pi]:$

$$
\begin{aligned}
& V_{\mathrm{r}}=-\Omega \mathrm{zr} / \mathrm{R} \\
& \mathrm{V}_{\theta}=0 \\
& \mathrm{~V}_{\mathrm{z}}=\frac{\Omega}{\mathrm{R}}\left(\mathrm{z}^{2}-\mathrm{H}^{2}\right)
\end{aligned}
$$

où $\Omega$ est un paramètre cinématique dont la signification apparaîtra clairement dans la suite. On observera la variation linéaire de la vitesse radiale en fonction de $z$, ce qui confère à la " déformée » (virtuelle) une géométrie de cône avec évasement vers le bas (voir fig. 8b).

Les valeurs propres de $\mathrm{d}$ sont :

$$
\begin{aligned}
& \mathrm{d}_{1}=-\frac{\Omega}{\mathrm{R}} \mathrm{z} \\
& \mathrm{d}_{2}=\frac{\Omega}{\mathrm{R}}\left(\frac{\left.\mathrm{z}-\sqrt{9 \mathrm{z}^{2}+\mathrm{r}^{2}}\right)}{2}\right) \\
& \mathrm{d}_{3}=\frac{\Omega}{\mathrm{R}}\left(\frac{\mathrm{z}+\sqrt{9 \mathrm{z}^{2}+\mathrm{r}^{2}}}{2}\right)
\end{aligned}
$$

Il résulte de (33) et (51) que :

$$
\pi(\underline{\underline{d}})=\frac{\Omega}{\mathrm{R}} \mathrm{C}_{r}\left(-\mathrm{z}+\sqrt{9 \mathrm{z}^{2}+\mathrm{r}^{2}}\right)
$$

puis, par intégration :

$$
\begin{aligned}
P_{\mathrm{r}} & =2 \pi \frac{\Omega}{\mathrm{R}} \mathrm{C}_{r} \int_{0}^{\mathrm{H}} \mathrm{dz} \int_{0}^{\mathrm{R}} \mathrm{r}\left(\mathrm{z}+\sqrt{9 \mathrm{z}^{2}+\mathrm{r}^{2}}\right) d r \\
& =2 \pi \frac{\Omega}{\mathrm{R}} \mathrm{C}_{\mathrm{r} g}(\mathrm{R}, \mathrm{H})
\end{aligned}
$$

où l'on a posé :

$g(\mathrm{R}, \mathrm{H})=\int_{0}^{\mathrm{H}} \mathrm{dz} \int_{0}^{\mathrm{R}} \mathrm{r}\left(\mathrm{z}+\sqrt{9 \mathrm{z}^{2}+\mathrm{r}^{2}}\right) \mathrm{dr}$

Le calcul de la fonction g est détaillée à l'annexe 1.

A nouveau, le choix $\mathrm{f}(-\mathrm{H})=0$ assure que la contribution de l'interface substratum-remblai à la puissance résistante maximale est nulle. Il s'agit maintenant de compléter la description du mécanisme dans l'enceinte de palplanches.

\section{- Cinématique des palplanches}

On définit les vitesses virtuelles dans l'enceinte de la manière suivante:

- le champ de vitesses dans chaque palplanche est rigidifiant ;

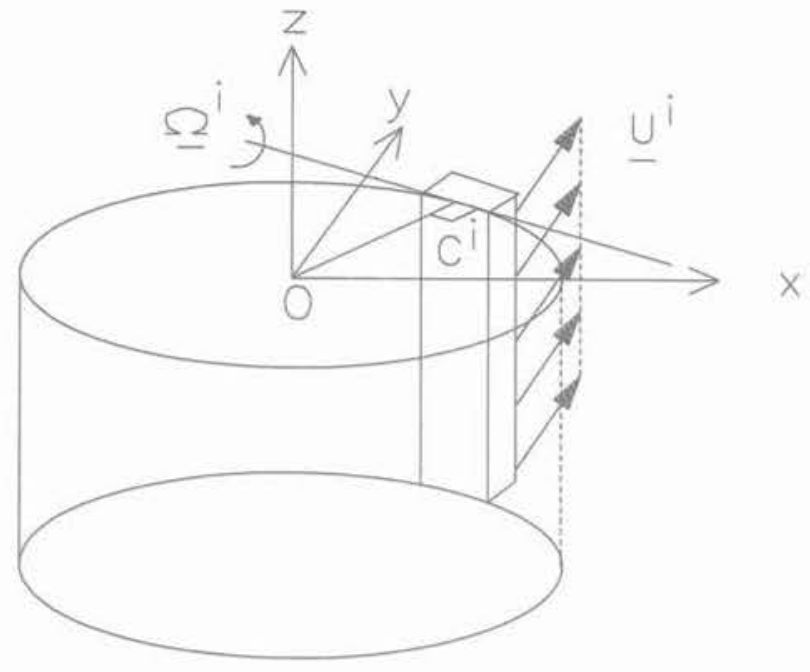

(a) palplanches

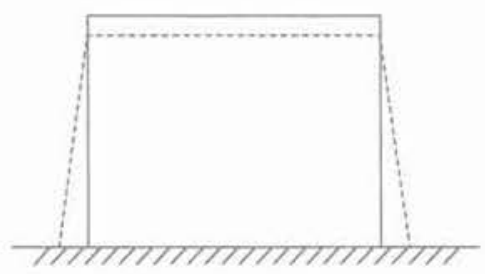

(b) remblai

Fig. 8. - Champ de vitesses virtuelles no2. Fig. 8, - Virtual velocity field $n^{\circ} 2$

- le champ de vitesses de la palplanche $n^{\circ} i$ (voir fig. 8a) est la composition d'une translation et d'une rotation autour de l'axe du plan $z=0$, tangent en $\mathrm{C}^{\prime}$ à la face interne de l'enceinte. La vitesse de rotation est donnée par :

$$
\underline{\Omega}^{1}=\Omega\left[\underline{e}_{x} \sin (2 \mathrm{i}-1) \frac{\pi}{\mathrm{n}}-\underline{\mathrm{e}}_{y} \cos (2 \mathrm{i}-1) \frac{\pi}{\mathrm{n}}\right]
$$

qui fournit l'interprétation physique du paramètre $\Omega$ intervenant également dans (50).

Le vecteur de la translation est défini par :

$$
\begin{aligned}
& \underline{U}^{i}=\Omega H \frac{1-\cos (\pi / n)}{\cos (\pi / n)}\left[\underline{e}_{x} \cos (2 i-1) \frac{\pi}{n}\right. \\
& \left.+\underline{e}_{y} \sin (2 i-1) \frac{\pi}{n}\right]+\left[\left(1-\cos \frac{\pi}{n}\right) \Omega R\right] \underline{e}_{z}
\end{aligned}
$$

Il s'agit à nouveau d'un mécanisme d'éclatement radial combiné de plus avec une cinématique de rotation et une translation verticale ascendante indépendante de la palplanche considérée. L'intensité du vecteur $\mathrm{U}^{\prime}$, comme celle du vecteur rotation $\Omega^{\prime}$ est choisie, 
comme on le verra plus loin, en sorte d'éviter l'interpénétration des palplanches avec le remblai et le substratum, c'est-à-dire pour assurer la positivité de la composante normale de la discontinuité de vitesses sur les interfaces remblai/palplanches et substratum/palplanches, condition nécessaire pour que la puissance résistance maximale soit finie. On calcule maintenant les contributions des différentes interfaces faisant intervenir les palplanches.

\section{- Contribution des palplanches à la puissance} résistance maximale

Interface palplanche-remblai

On calcule la vitesse d'un point $\mathrm{M}$ situé sur la face interne de la palplanche $\mathrm{n}^{\circ} \mathrm{i}$, de coordonnées cylindriques $\left(\mathrm{R},(2 \mathrm{i}-1) \frac{\pi}{\mathrm{n}}+\delta, \mathrm{z}\right)$ :

$$
\begin{aligned}
\underline{\mathrm{OM}} & =\mathrm{R}\left[\underline{\mathrm{e}}_{\mathrm{x}} \cos \left\{(2 \mathrm{i}-1) \frac{\pi}{\mathrm{n}}+\delta\right\}\right. \\
& \left.+\underline{e}_{y} \sin \left\{(2 \mathrm{i}-1) \frac{\pi}{\mathrm{n}}+\delta\right\}\right]+z\left(\underline{e}_{y}\right)^{(57)}
\end{aligned}
$$

avec $\delta \in\left[-\frac{\pi}{n},+\frac{\pi}{n}\right]$ et $z \in[-H, 0]$. L'expression de la vitesse du point $\mathrm{M}$ dans le mouvement de la palplanche $n^{\circ} i$ est alors :

$$
\underline{\mathrm{V}}^{\mathrm{p}}=\underline{\mathrm{U}}^{\mathrm{i}}+\underline{\Omega}^{i} \wedge \underline{\mathrm{C}^{i} \mathrm{M}}
$$

avec :

$$
\begin{aligned}
\underline{O C^{\prime}} & =R\left(\underline{e}_{x} \cos \left\{(2 i-1) \frac{\pi}{n}\right\}\right. \\
& \left.+\underline{e}_{y} \sin \left\{(2 i-1) \frac{\pi}{n}\right\}\right)
\end{aligned}
$$

soit encore, après calcul :

$$
\begin{aligned}
\underline{\mathrm{V}}^{\mathrm{P}} & =-2 \Omega\left(\underline{e}_{\mathrm{x}} \cos \left\{(2 \mathrm{i}-1) \frac{\pi}{\mathrm{n}}\right\}\right. \\
& \left.+\underline{e}_{y} \sin \left\{(2 \mathrm{i}-1) \frac{\pi}{\mathrm{n}}\right\}\right) \\
& +\underline{e}_{2} \Omega \mathrm{R}(\cos \delta-1)+\underline{U}^{i}
\end{aligned}
$$

L'expression de la vitesse du point $M$ dans le mouvement du remblai est :

$$
\begin{aligned}
\underline{\mathrm{V}}^{T} & =-z \Omega\left(\underline{e}_{\mathrm{x}} \cos \left\{(2 \mathrm{i}-1) \frac{\pi}{\mathrm{n}}+\delta\right\}\right. \\
& \left.+\underline{e}_{y} \sin \left\{(2 \mathrm{i}-1) \frac{\pi}{\mathrm{n}}+\delta\right\}\right) \\
& +\underline{e}_{z} \frac{\Omega}{\mathrm{R}}\left(\mathrm{z}^{2}-\mathrm{H}^{2}\right)
\end{aligned}
$$

Pour vérifier que la contribution de cette interface à la puissance résistante maximale est finie, il est nécessaire et suffisant de vérifier que le produit scalaire $\left(\mathrm{V}^{\mathrm{p}}-\mathrm{V}^{\mathrm{V}}\right)$. er est positif ou nul pour toute valeur de $z \in[-H, 0]$ et $\delta \in\left[-\frac{\pi}{n},+\frac{\pi}{n}\right]$. Il est facile de voir que :

$$
\begin{aligned}
& \left.\underline{\mathrm{V}}^{\mathrm{P}}-\underline{\mathrm{V}}^{\mathrm{N}}\right) \cdot \underline{e}_{\underline{y}}=\Omega \mathrm{H} \frac{1-\cos (\pi / \mathrm{n})}{\cos (\pi / \mathrm{n})} \cos \delta \\
& +\Omega z(1-\cos \delta)
\end{aligned}
$$

Il suffit de vérifier la propriété annoncée pour $\mathrm{z}=-\mathrm{H}$, (puisque $1-\cos \delta>0$ ). On est donc ramené à étudier le signe de la quantité

$$
\begin{aligned}
& \Omega H \frac{1-\cos (\pi / n)}{\cos (\pi / n)} \cos \delta-\Omega H(1-\cos \delta) \\
& =\frac{\cos \delta}{\cos (\pi / n)}-1
\end{aligned}
$$

Il est immédiat de vérifier que cette quantité est toujours $\geq 0$ dans $\left[-\frac{\pi}{n},+\frac{\pi}{n}\right]$ et s'annule aux bornes de cet intervalle. Il en résulte que la contribution de l'interface étudié est nulle.

\section{Interface palplanche-substratum}

On considère un point $\mathrm{M}$ de l'interface entre la palplanche $n^{\circ} i$ et le substratum, de coordonnées cylindriques $\left(r,(2 i-1) \frac{\pi}{n}+\delta,-H\right)$ avec $r \in[R, R+e]$ et $\delta \in\left[-\frac{\pi}{n},+\frac{\pi}{n}\right]$. En observant que la discontinuité vitesse de continuité au niveau de cette interface coïncide avec la vitesse $\mathrm{V}^{\mathrm{P}}(\mathrm{M})$ du point $\mathrm{M}$ dans le mouvement de la palplanche $n^{\circ} i$, il est facile de vérifier que la composante normale à l'interface considérée de la discontinuité de vitesse vaut:

$$
\begin{aligned}
& \underline{\mathrm{V}}^{\mathrm{P}}(\mathrm{M}) \cdot \underline{e}_{3}=\Omega\left[\mathrm{R}\left(\cos \delta-\cos \frac{\pi}{\mathrm{n}}\right)+\right. \\
& (\mathrm{r}-\mathrm{R}) \cos \delta] \geq 0
\end{aligned}
$$

La contribution correspondante à la puissance résistante maximale est donc nulle.

Interfaces entre palplanches

Il s'agit d'abord de calculer les vitesses d'un point $\mathrm{M}$ de l'interface entre les palplanches $n^{\circ} i$ et $i+1$ dans le mouvement respectif de ces palplanches. Les coordonnées cylindriques d'un tel point sont $\left(r, \theta=2 i \frac{\pi}{n}\right.$, z) avec $r \in[R, R+e], z \in[-H, 0]$.

On note $\underline{V}^{i}$ la vitesse du point $M$ dans le mouvement de la palplanche $\mathrm{n}^{0} \mathrm{i}$ :

$$
\begin{aligned}
& \underline{V}^{i}=-2 \Omega\left(\underline{e}_{x} \cos \left((2 \mathrm{i}-1) \frac{\pi}{\mathrm{n}}\right\}\right. \\
& \left.+\underline{e}_{y} \sin \left\{(2 \mathrm{i}-1) \frac{\pi}{\mathrm{n}}\right\}\right) \\
& +\underline{e}_{2} \Omega(\mathrm{r} \cos \delta-\mathrm{R})+\underline{\mathrm{U}}^{\mathrm{i}}
\end{aligned}
$$


On note $\underline{V}^{i+1}$ la vitesse du point $M$ dans le mouvement de la palplanche $n^{\circ} \mathrm{i}+1$ :

$$
\begin{aligned}
& \underline{V}^{i+1}=-2 \Omega\left(\underline{e}_{x} \cos \left\{(2 i+1) \frac{\pi}{n}\right\}\right. \\
& \left.+\underline{e}_{y} \sin \left\{(2 i+1) \frac{\pi}{n}\right\}\right) \\
& +\underline{e}_{z} \Omega\left(r \cos \frac{\pi}{n}-R\right)+\underline{U}^{i+1}
\end{aligned}
$$

Posant $[\underline{V}]=\underline{V}^{i+1}-\underline{V}^{i}$, il vient:

$$
\begin{aligned}
& {[\underline{V}]=2 \Omega \sin \left(\frac{\pi}{n}\right)\left(H \frac{1-\cos (\pi / n)}{\cos (\pi / n)}-z\right)} \\
& \left(-\underline{e}_{x} \sin 2 i \frac{\pi}{n}+\underline{e}_{y} \cos 2 i \frac{\pi}{n}\right)
\end{aligned}
$$

Il apparaît donc que la discontinuité de vitesse au niveau des interfaces entre palplanches est purement orthoradiale, orientée dans le sens de l'éloignement des palplanches, Il est alors facile de voir que la contribution $P_{p}$ des $n$ interfaces entre palplanches se calcule par:

$P_{p}=n \int_{-H}^{0} d z \int_{R}^{R+e} d r \cdot\left(T_{0} / e\right) \cdot\|[\underline{V}]\|=$

$2 n \Omega \sin \left(\frac{\pi}{n}\right) T_{0} \int_{-H}^{0}\left(H \frac{1-\cos (\pi / n)}{\cos (\pi / n)}-z\right) d z$

soit finalement :

$P_{p}=T_{0} \operatorname{\Omega nsin}\left(\frac{\pi}{n}\right) H^{2}\left(1+2 \frac{1-\cos (\pi / n)}{\cos (\pi / n)}\right)$

\section{- Application du théorème cinématique}

L'emploi du théorème cinématique requiert le calcul de la puissance du poids du remblai. Notant $\mathbb{P}_{\text {ext }}$ cette quantité, il vient :

$$
P_{\text {ext }}=\gamma_{t} \int_{0}^{R} d r \int_{0}^{2 \pi} r d \theta \int_{-H}^{0} \frac{\Omega}{R}\left(H^{2}-z^{2}\right) d z
$$

soit :

$$
\rho_{\text {ext }}=\frac{2}{3} \pi \gamma_{\mathrm{r}} \Omega \mathrm{RH}^{3}
$$

à laquelle il conviendrait en toute rigueur d'ajouter la contribution (résistante) du poids des palplanches à la puissance des forces extérieures qui pourra cependant être négligée dans le cadre des hypothèses $n \gg 1$ et $\mathrm{R} / \mathrm{e} \gg 1$. Il résulte de (53), (69) et (71) une condition nécessaire de stabilité sous la forme suivante :

$$
\begin{aligned}
& \frac{2}{3} \pi \gamma_{\mathrm{r}} \Omega \mathrm{RH}^{3} \leq \Omega \pi \mathrm{T}_{0} \mathrm{H}^{2} \\
& +2 \pi \mathrm{C}_{\mathrm{r}} \frac{\Omega \mathrm{H}^{4}}{\mathrm{R}}\left(\mathrm{g} / \mathrm{H}^{4}\right)
\end{aligned}
$$

où l'on a supposé (dans l'expression de $P_{p}$ ) que $\lim _{n \rightarrow \infty} P_{D}$ constitue une bonne approximation de $\mathrm{P}_{\mathrm{p}}$. Après simplification, on obtient la condition nécessaire de stabilité suivante :

$$
k \leq \frac{3}{2}\left(T_{0} / R C\right)+3\left(g / H^{4}\right)(H / R)^{2}
$$

\section{- Application numérique}

En combinant les inégalités (24b) et (73), on met en évidence un encadrement du rapport adimensionnel limite $\mathrm{k}^{+}$. Avec les valeurs numériques du rapport $4 / \mathrm{H}^{4}$ données à l'annexe 1 pour les élancements $\mathrm{H} / \mathrm{R}=1,2$ et 3 , on obtient :

$$
\begin{gathered}
\left(\mathrm{T}_{0} / \mathrm{RC}_{\mathrm{r}}\right)+2 \leq \mathrm{k}^{+} \leq \\
\frac{3}{2}\left(\mathrm{~T}_{0} / \mathrm{RC}_{\mathrm{r}}\right)+\left\{\begin{array}{l}
3,318 \text { pour } \mathrm{H} / \mathrm{R}=1 \\
3,096 \text { pour } \mathrm{H} / \mathrm{R}=2 \\
3,05 \text { pour } \mathrm{H} / \mathrm{R}=3
\end{array}\right.
\end{gathered}
$$

On peut montrer que :

$$
\lim _{\mathrm{R} / \mathrm{H} \rightarrow 0}\left(g / \mathrm{H}^{4}\right) \cdot(\mathrm{H} / \mathrm{R})^{2}=1
$$

ce qui signifie que le rapport entre les majorant et minorant de $\mathrm{k}^{+}$tend vers $3 / 2$ lorsque $\mathrm{R} / \mathrm{H}$ tend vers 0 . Pour les valeurs usuelles de $R / H$, ces quantités sont dans un rapport proche de $3 / 2$.

\section{CELLULE A REMBLAI EN MATÉRIAU FLOTTANT}

\subsection{Approche statique}

Les conditions définissant les champs de contraintes statiquement admissibles ont été définies en (12) et (13). On recherche à nouveau un tel champ de contraintes pour lequel les directions verticale, radiale et orthoradiale soient principales et qui soit compatible avec les capacités de résistance décrite en 3.3 , en adoptant le critère (6) pour le matériau de remblai. En vertu de l'argumentation présentée en 4.1. l'approche statique peut être limitée à la cellule. On examine à quelle condition sur $\gamma_{r}$ et $\gamma_{a}$ le champ de contrainte défini ci-dessous en fonction d'un paramètre scalaire positif $k$ répond à la question :

- remblai :

$$
\begin{aligned}
& \underline{\underline{\sigma}}=\gamma_{\mathrm{r}} z\left\{\left[\underline{e}_{\mathrm{S}} \otimes \underline{\mathrm{e}}_{\mathrm{s}}+\underline{\mathrm{e}}_{\theta} \otimes \underline{e}_{\theta}\right]+\underline{e}_{2} \otimes \underline{e}_{2}\right\} \\
& \text { - enceinte: } \\
& \underline{\underline{\sigma}}=\left[\mathrm{a}(\mathrm{z})+\mathrm{b}(\mathrm{z}) / \mathrm{r}^{2}\right] \underline{e}_{\mathrm{e}} \otimes \underline{e}_{\mathrm{s}}+ \\
& {\left[a(z)-b(z) / r^{2}\right] \underline{e}_{\theta} \otimes \underline{e}_{\theta}+\left(\gamma_{a} z\right) \underline{e}_{z} \otimes \underline{e}_{z}}
\end{aligned}
$$


Les équations de champ (12a) et (12b) sont manifestement satisfaites, ainsi que la condition aux limites (13b). La valeur des fonctions $a(z)$ et $b(z)$ est déterminée en écrivant la continuité du vecteurcontrainte à l'interface remblai/palplanches et la condition aux limites (13a) en $r=R+e$ :

$$
\begin{aligned}
& \mathrm{a}(\mathrm{z})=\frac{-\mathrm{b}(\mathrm{z})}{(\mathrm{R}+\mathrm{e})^{2}} \\
& \mathrm{~b}(\mathrm{z})=\frac{\gamma_{\mathrm{r}} \mathrm{kz}}{1 / \mathrm{R}^{2}-1(\mathrm{R}+\mathrm{e})}
\end{aligned}
$$

qui déterminent un champ s.a. avec $\gamma_{\mathrm{r}}$ et $\gamma_{\mathrm{a}}$. Le scalaire k étant positif, le critère d'interface $\mathrm{r} / \mathrm{p}$ est respecté. Il en est de même des interfaces $\mathrm{p} / \mathrm{s}$ et $\mathrm{r} / \mathrm{s}$, en raison du signe négatif de $\sigma_{\mathrm{zz}}$. Pour que le champ $\sigma$ défini par (76). (77a) et (77b) soit compatible avêc les capacités de résistance, il reste à vérifier les critères $f_{p}(\underline{T})$ et $f_{\mathrm{r}}(\underline{\underline{\sigma}})$.

\section{Critère $f_{p}(T)$ :}

la condition de compatibilité du champ de contraintes $\underline{\sigma}$ avec le critère $\mathrm{f}_{\mathrm{p}}(\mathrm{T})$ a été exprimée en (23). Elle s'écrit ici :

$$
\begin{aligned}
& (\forall r \in[R, R+e])(\forall z \in[-H, 0]) \\
& |b(z)|\left\{\frac{1}{(R+e)^{2}}+\frac{1}{r^{2}}\right] \leq T_{0} / e
\end{aligned}
$$

soit encore :

$$
\gamma_{r} R H\left\{\frac{1 / \mathrm{R}^{2}+1 /(\mathrm{R}+e)^{2}}{1 / \mathrm{R}^{2}-1 /(\mathrm{R}+e)^{2}}\right\} \leq \mathrm{T}_{0} / e
$$

ou, en négligeant le premier ordre en $\mathrm{e} / \mathrm{R}$.

$$
\gamma_{\mathrm{r}} \leq \frac{\mathrm{T}_{\mathrm{D}}}{\mathrm{RHH}}
$$

Critère $f_{r}(\underline{\underline{g}})$ :

La forme donnée en (76) pour $\underline{\sigma}$ est compatible avec le critère $f_{\mathrm{r}}(\sigma)$ défini en (6) si et seulement si :

$$
k \in\left[\mathrm{K}_{\mathrm{a}}, \mathrm{K}_{\mathrm{p}}\right]
$$

où $K_{a}$ et $K_{p}$ désignent les coefficients de poussée et de butée que l'on calcule à partir de $\phi_{\mathrm{r}}$ selon les relations :

$$
\mathrm{K}_{\mathrm{a}}=1 / \mathrm{K}_{\mathrm{p}}=\frac{1-\sin \phi_{\mathrm{r}}}{1+\sin \phi_{\mathrm{r}}}
$$

En comparant (80) et (81), il apparait que $\mathrm{K}_{\mathrm{p}} \mathrm{T}_{0} /(\mathrm{RH})$ représente la plus grande valeur de $\gamma_{\mathrm{T}}$ qu'il soit possible d'équilibrer par un champ de type (76)-(77) en sorte que ce champ soit compatible avec les capacités de résistance de la structure. Il en résulte que :

$$
\gamma_{\mathrm{r}}^{+} \geq \frac{1}{\mathrm{~K}_{\mathrm{a}}} \frac{\mathrm{T}_{\mathrm{o}}}{\mathrm{RH}}=\mathrm{K}_{\mathrm{p}} \frac{\mathrm{T}_{\mathrm{o}}}{\mathrm{RH}}
$$

\subsection{Retour aux méthodes de dimensionnement classiques}

Le dimensionnement classique conduit à introduire, comme on l'a indiqué au paragraphe 2.2., un concept de poids volumique maximal supportable, défini par la relation $\gamma_{i}^{*}=\frac{1}{\mathrm{~K}}\left(\mathrm{~T}_{0} / \mathrm{RH}\right)$. On a souligné précédemment la faiblesse de cette notion, en l'absence d'une procédure de détermination du coefficient $\mathrm{K}$. La théorie du calcul à la rupture permet d'identifier l'origine de cette difficulté.

En effet, dans le cas où les informations disponibles sur le(s) matériau(x) se limitent à la donnée des capacités de résistance, elle rappelle que seule la borne supérieure $\gamma_{r}^{+}$des valeurs potentiellement supportables du poids volumique, au sens défini en 2.1 ., peut être déterminée ou encadrée. En dehors de la donnée d'informations complémentaires, il est donc vain de rechercher la valeur du poids volumique maximal. C'est la raison pour laquelle le choix de $\mathrm{K}$ dans l'expression de $\mathrm{F}$ ou de $\gamma_{\mathrm{r}}^{*}$ est nécessairement arbitraire ou empirique. Cependant, l'analogie entre $\gamma_{\mathrm{r}}^{*}$ et la formule (83) ci-dessus appelle quelques commentaires.

Dans le cas du remblai purement frottant, lanalyse dimensionnelle du problème de stabilité indique que le paramètre adimensionnel $\gamma_{\mathrm{r}}^{+} \mathrm{HR} / \mathrm{T}_{0}$ est une fonction du rapport $\mathrm{R} / \mathrm{H}$, ce qui amène à rechercher la fonction $\mathcal{F}$ telle que :

$$
\frac{\gamma_{\mathrm{r}}^{+} \mathrm{HR}}{\mathrm{T}_{0}}=\mathcal{F}\left(\frac{\mathrm{R}}{\mathrm{H}}\right)
$$

A partir d'hypothèses formulées sur la structure du champ de contraintes dans la cellule à la rupture, le dimensionnement classique revient donc à postuler que $\mathcal{F}$ est une fonction constante, dont la valeur est déterminée empiriquement où arbitrairement. Partant de l'hypothèse que le remblai est, à la rupture de la cellule, dans l'état de poussée limite, certains auteurs (par exemple SCHNEEBELl et al., 1957) proposent de retenir la valeur $\mathrm{K}=\mathrm{K}_{\mathrm{a}}$ dans l'expression de $\gamma_{\mathrm{r}}^{*}$; c'est-à-dire $F=1 / K_{a}$. L'approche statique du calcul à la rupture, telle qu'elle a été développée au paragraphe 5.1. conduit à un minorant de la fonction $\mathcal{F}(\mathrm{R} / \mathrm{H})$ qui n'est autre que $1 / \mathrm{K}_{\mathrm{a}}$. L'expression de $\gamma_{i}$ coïncide alors avec le minorant de $\gamma_{r}^{+}$donné en (83). La différence de nature entre les démarches conduisant respectivement à $\gamma_{\mathrm{r}}^{*}$ et (83) démontre pourtant le caractère purement formel de lanalogie observée. En effet, l'approche développée au paragraphe 5.1. a consisté à examiner, dans lespace des champs de contraintes statiquement admissibles avec $\gamma_{\mathrm{r}}$, un sous-ensemble défini par (76), et à optimiser le choix du paramètre $k y$ intervenant. C'est ce processus d'optimisation qui a conduit à $k=\mathrm{K}_{a}$, sans qu'il soit nécessaire de faire quelque hypothèse que ce soit sur l'état de contraintes à la rupture.

Il importe enfin de souligner que (83) est, non pas une égalité, mais une inégalité. En d'autres termes, $\mathrm{T}_{\mathrm{o}} / \mathrm{RHK}_{\mathrm{a}}$ n'est qu'un minorant du poids volumique extrême. La proposition $\gamma_{\mathrm{r}} \leq \mathrm{T}_{0} / \mathrm{RHK}_{\mathrm{a}}$ est donc une condition suffisante de stabilité potentielle, alors 
que lapproche traditionnelle de SCHNEEBELI et al. (1957) présente la même inégalité $\gamma_{\mathrm{r}} \leq \mathrm{T}_{0} / \mathrm{RHK}_{\mathrm{a}}$ comme une condition nécessaire pour que la stabilité puisse être assurée.

\subsection{Approche cinématique}

On reprend, tant pour le remblai, que pour l'enceinte de palplanches, le mécanisme de transformation homogène décrit et utilisé dans le cas de la structure " cohérente . On pose donc :

$$
\begin{aligned}
& V_{r}=\lambda r \\
& V_{\theta}=0 \\
& V_{z}=\lambda^{\prime}(z+H)
\end{aligned}
$$

Cependant, la condition $\pi$ (d) $<+\infty$ impose à la place de (28) la relation suivante entre $\lambda$ et $\lambda^{\prime}$ :

$$
2 \lambda+\lambda^{\prime} \geq\left(2|\lambda|+\left|\lambda^{\prime}\right|\right) \sin \phi_{\mathrm{r}}
$$

Pour que les forces de gravité soient motrices, il est nécessaire de prendre $\lambda^{\prime}$ négatif. Le scalaire $\lambda$ est donc positif et la condition (86) peut-être réécrite sous la forme d'une égalité :

$$
\begin{aligned}
& \lambda^{\prime}=-2 \mathrm{~K}_{\mathrm{a}} \theta \lambda ; \theta \in[0,1] ; \\
& \mathrm{K}_{\mathrm{a}}=\frac{1-\sin \phi_{\mathrm{r}}}{1+\sin \phi_{\mathrm{r}}}
\end{aligned}
$$

Pour un mécanisme de type (85) vérifiant de plus (87), la contribution du remblai à la puissance résistante maximale est nulle:

$$
P_{r}=0
$$

La cinématique de lenceinte de palplanches est inchangée. La contribution des interfaces entre palplanches a été donnée en (45). Enfin, en reprenant le calcul exposé en (46) on trouve:

$$
\theta_{\text {ext }}=\gamma_{t} \pi \mathrm{R}^{2} \mathrm{H}^{2} \lambda \theta \mathrm{K}_{\mathrm{a}}
$$

La condition de stabilité fournie par le théorème cinématique prend donc la forme suivante:

( $\forall \theta \in[0,1]) \lambda_{\gamma} \pi \mathrm{R}^{2} \mathrm{H}^{2} \theta \mathrm{K}_{\mathrm{a}} \leq 2 \pi \lambda R H T_{0}$

L'inégalité la plus restrictive est obtenue pour $\theta=1$ :

$$
\gamma_{\mathrm{r}}^{+} \leq 2 \mathrm{~K}_{\mathrm{p}} \mathrm{T}_{0} / \mathrm{RH}
$$

Le fait que le majorant de $\gamma_{\mathrm{r}}^{+}$donné en (91) soit une fonction croissante de $T_{0}$ et $\phi_{\mathrm{r}}$, et une fonction décroissante de $\mathrm{H}$ correspond à l'intuition naturelle. La décroissance par rapport à $\mathrm{R}$ s'explique par le fait que les contraintes d'arrachement au niveau des interfaces entre palplanches sont une fonction croissante du rayon de la cellule.

\section{CONCLUSION}

L'analyse de la stabilité d'une cellule élémentaire de gabion posée sur un substratum rigide et soumise aux seules forces de gravité a été développée dans le cadre de la théorie du calcul à la rupture. On a supposé que les interfaces entre le remblai, les palplanches et le substratum étaient lisses et sans résistance à la traction. La résistance à l'arrachement des palplanches a été prise en compte dans le cadre d'un critère d'interface au niveau des serrures. Deux développements distincts ont été proposés, respectivement dans le cas d'un matériau de remblai purement cohérent et dans le cas où il est purement frottant.

Dans le cas du matériau cohérent de cohésion $C_{r}$, l'analyse dimensionnelle fait intervenir les 2 paramètres adimensionnels $\mathrm{k}=\gamma_{\mathrm{r}} \mathrm{H} / \mathrm{C}_{\mathrm{r}}$ et $\mathrm{T}_{0} /\left(\mathrm{RC}_{\mathrm{r}}\right)$. La combinaison des approches statique et cinématique montre alors que $\frac{5}{4}\left(2+\mathrm{T}_{0} /\left(\mathrm{RC}_{\mathrm{\gamma}}\right)\right)$ constitue une estimation du paramètre de chargement extrême $\mathrm{k}^{+}$ à moins de $25 \%$ d'erreur près.

Il est instructif d'exprimer ce résultat sous une forme faisant apparaître le poids volumique extrême $\gamma_{f}^{+}$:

$$
\gamma_{r}^{+} \cong \frac{5}{4}\left(2 \mathrm{C}_{\mathrm{r}} / \mathrm{H}+\mathrm{T}_{0} /(\mathrm{RH})\right)
$$

quî met en évidence les rôles additifs joués par la cohésion et la résistance à l'arrachement dans la tenue de l'ouvrage. On observera notamment que la cohésion devient essentielle à la tenue de la cellule pour les grandes valeurs du rayon.

Cette relation peut également être utilisée en sens inverse, c'est-à-dire qu'elle permet de déterminer les dimensions d'une cellule pour des valeurs connues des capacités de résistance et du poids volumique.

Si lon considère maintenant le matériau frottant, un encadrement du poids volumique extrême a pu être établi :

$$
\mathrm{K}_{\mathrm{p}} \mathrm{T}_{0} / \mathrm{RH} \leq \gamma_{\mathrm{r}}^{+} \leq 2 \mathrm{~K}_{\mathrm{p}} \mathrm{T}_{0} / \mathrm{RH}
$$

Issu d'une combinaison d'approches statique et cinématique très simples, il reste relativement imprécis. Il présente néanmoins lavantage de mettre en évidence l'importance du groupement $\mathrm{K}_{\mathrm{p}} \mathrm{T}_{0} / \mathrm{RH}$, minorant du poids volumique extrême.

En conclusion, la présente étude propose une analyse mécanique tridimensionnelle de la question de la stabilité d'une cellule élémentaire posée sur un substratum rigide et soumise à son poids propre. Elle permet une relecture des méthodes classiques d'analyse de la stabilité interne en précisant notamment le caractère nécessaire ou suffisant des conditions de stabilité obtenues. L'intérêt de la démarche adoptée, c'est-àdire le calcul à la rupture, réside aussi dans le fait qu'elle peut être étendue aisément à une cellule fichée dans un massif ayant des caractéristiques de résistance bornées. La prise en compte de sollicitations plus complexes pourra également être traitée dans le même esprit en s'appuyant essentiellement sur l'approche cinématique. 


\section{ANNEXE 1}

\section{Rappelons que :}

$g(R, H)=\int_{0}^{H} d z \int_{0}^{R} r\left(z+\sqrt{9 z^{2}+r^{2}}\right) d r$

Le calcul de l'intégrale $g(R, H)$ donne:

$y=\frac{H^{4}}{4} \cdot\left\{\left(\frac{R}{H}\right)^{2}+\frac{4}{3}\left(\frac{R}{H}\right)^{4} g\left(\frac{H}{R}\right)-9\right\}$

la fonction $\mathrm{g}(\mathrm{x})$ étant définie par : $g(x)=\int_{0}^{x}\left(1+9 u^{2}\right)^{3 / 2}$ du. On observe que le rapport $\mathrm{g} / \mathrm{H}^{4}$ ne dépend que du rapport $\mathrm{R} / \mathrm{H}$. A l'aide du changement de variables $\mathrm{z}^{2} \mathrm{u}^{2}=1+9 \mathrm{u}^{2}$, on peut voir que :

$$
\int\left(1+9 u^{2}\right)^{3 / 2} d u=-\int \frac{z^{4}}{\left(z^{2}-9\right)^{3}} d z
$$

Puis l'on montre que :

$$
\begin{aligned}
& -\int \frac{z^{4}}{\left(z^{2}-9\right)^{3}} d z=\frac{5 z}{8\left(z^{2}-9\right)} \\
& +\frac{9 z}{4\left(z^{2}-9\right)^{2}}-\frac{1}{16} \ln \left(\frac{z-3}{z+3}\right)
\end{aligned}
$$

Notant $F(z)$ la fonction définie ci-dessus, il vient finalement :

$$
\begin{aligned}
& g(x)=-\int_{\infty}^{\sqrt{9+1 / x^{2}}} \frac{z^{4}}{\left(z^{2}-9\right)^{3}} d z \\
& =F\left(\sqrt{\left.9+1 / x^{2}\right)}\right.
\end{aligned}
$$

Le calcul numérique fournit :

$\mathrm{g}(1)=9,319 ; \mathrm{g}(2)=117,4 ; \mathrm{g}(3)=567,44$ dont on déduit les valeurs numériques suivantes du rapport $\mathrm{g} / \mathrm{H}^{4}$ :

pour $\mathrm{H} / \mathrm{R}=1: \mathrm{g} / \mathrm{H}^{4}=1,106$

pour $H / R=2: g / H^{4}=0,258$

pour $\mathrm{H} / \mathrm{R}=3: \mathrm{g} / \mathrm{H}^{4}=0,113$

\section{REMERCIEMENTS}

Ce travail a été développé dans le cadre d'une collaboration avec le Service Technique Central des Ports Maritimes et des Voies Navigables. Les auteurs tiennent également à remercier $\mathrm{P}$. DE BUHAN pour l'aide qu'il leur a apportée dans la réalisation de cette étude.

\section{BIBLIOGRAPHIE}

CLOUGH G., MOSHER R., SINGH Y., KUPUSAMY T. (1987), Etude des batardeaux cellulaires par une modélisation aux éléments finis en deux et trois dimensions. Actes du colloque Interactions Sols-Structures, Paris, 1987 , pp. 553-560.

LACROIX Y., ESRIG M.I., LUSCHER U. (1970), Design, construction and performance of cellular cofferdams. Lateral Stresses in the Ground and Design of Earth-Retaining Structures, ASCE Specialty Conference, pp. 271-328.

NAVDOCKS DM-7.02 (1986), Design manual, foundations and earth structures. Naval Facilities Engineering Command.

SALENÇON J. (1983), Calcul à la rupture et analyse limite. Presses de l'Ecole Nationale des Ponts et Chaussées.

SALENÇON J. (1990), An introduction to the yield design theory and its applications to soil mechanics. European Journal of Mechanics, A/Solids, vol. $9, n^{\circ} 5$

SCHNEEBELI G., CAVAILLÉ-COLL R. (1957), Contribution to the stability analysis of double wall sheet pile cofferdams. Proceedings of the IV ${ }^{\text {th }}$ International Conference on soil mechanics and foundation engineering, London, 1957, pp. 233-238.

VERGOBBI M. (1979), Dimensionnement et calculs des gabions. Projet de fin d'études de l'ENPC. 